\title{
A broad-spectrum lipidomics screen of antiinflammatory drug combinations in human blood
}

\author{
Liudmila L. Mazaleuskaya, John A. Lawson, Xuanwen Li, Gregory Grant, Clementina Mesaros, \\ Tilo Grosser, Ian A. Blair, Emanuela Ricciotti, and Garret A. FitzGerald
}

Department of Systems Pharmacology and Translational Therapeutics and Institute for Translational Medicine and Therapeutics, Perelman School of Medicine, University of Pennsylvania, Philadelphia, Pennsylvania, USA.

\begin{abstract}
Current methods of drug screening in human blood focus on the immediate products of the affected pathway and mostly rely on approaches that lack sensitivity and the capacity for multiplex analysis. We have developed a sensitive and selective method based on ultra-performance liquid chromatography-tandem mass spectrometry to scan the effect of drugs on the bioactive eicosanoid lipidome in vitro and ex vivo. Using small sample sizes, we can reproducibly measure a broad spectrum of eicosanoids in human blood and capture drug-induced substrate rediversion and unexpected shifts in product formation. Microsomal prostaglandin E synthase-1 (mPGES-1) is an antiinflammatory drug target alternative to COX-1/-2. Contrasting effects of targeting mPGES-1 versus COX-1/-2, due to differential substrate shifts across the lipidome, were observed and can be used to rationalize and evaluate drug combinations. Finally, the in vitro results were extrapolated to ex vivo studies by administration of the COX- 2 inhibitor, celecoxib, to volunteers, illustrating how this approach can be used to integrate preclinical and clinical studies during drug development.
\end{abstract}

Conflict of interest: The authors have declared that no conflict of interest exists.

Submitted: February 11, 2016 Accepted: July 7, 2016 Published: August 4, 2016

Reference information: JCI Insight. 2016;1(12):e87031. doi:10.1172/ji.insight.87031.

\section{Introduction}

NSAIDs are among the most commonly prescribed drugs. They include aspirin, used in low doses for cardioprotection, and drugs such as ibuprofen and celecoxib, used for the relief of pain and inflammation (1). They target the prostaglandin (PG) G/H synthase enzymes, colloquially known as COXs (COX-1 and COX-2). Constitutively expressed COX-1 accounts largely for formation of PGs subserving "housekeeping" functions, such as thromboxane $\mathrm{A}_{2}\left(\mathrm{TxA}_{2}\right)$ in hemostasis and $\mathrm{PGE}_{2}$ and $\mathrm{PGI}_{2}$ in the maintenance of gastroduodenal epithelial integrity. Platelets express only COX-1, and suppression of platelet COX-1-dependent $\mathrm{TxA}_{2}$ by low-dose aspirin accounts for its cardioprotective effects (2). COX-2 is more readily regulated in its expression, especially by mitogens and cytokines, and accounts largely for elaboration of the $\mathrm{PGE}_{2}$ and $\mathrm{PGI}_{2}$ that mediate pain and inflammation. While some older NSAIDs, like ibuprofen, inhibit both COX-1 and COX-2, a series of COX-2-selective NSAIDs, including celecoxib, valdecoxib, and rofecoxib, were developed and brought to market. It was hoped that they would conserve the efficacy of mixed inhibitors while minimizing gastropathy, which was largely attributed to inhibition by NSAIDs of housekeeping PGs formed by COX-1. Indeed, the results of controlled trials ultimately supported this notion (3).

While suppression of COX-2-derived $\mathrm{PGE}_{2}$ and $\mathrm{PGI}_{2}$ explained the analgesic and antiinflammatory efficacy of these newer drugs, basic and clinical experimentation revealed that these same PGs, particularly $\mathrm{PGI}_{2}$, now formed in the cardiovascular system, afforded protective functions. Randomized trials ultimately demonstrated that COX-2-selective NSAIDs conferred a cardiovascular hazard, and rofecoxib and valdecoxib were withdrawn from the market (3). These discoveries shifted interest downstream in the PG biosynthetic pathway to the microsomal prostaglandin E synthase-1 (mPGES-1), the major source of $\mathrm{PGE}_{2}$ formation, as an alternative drug target. Here, studies in mice suggest that, rather than conferring cardiovascular risk by suppressing $\mathrm{PGI}_{2}$, inhibition of mPGES-1 might avoid this hazard or even confer cardiovascular benefit due to a shift of the $\mathrm{PGH}_{2}$ substrate to $\mathrm{PGI}_{2}$ synthase (Figure 1), augmenting $\mathrm{PGI}_{2}$ biosynthesis (4).

PGs and other products of arachidonic acid (AA) metabolism are formed in pM to fM amounts, acting locally as evanescent mediators (1). Furthermore, the capacity of tissues or cells to form these compounds, collectively termed eicosanoids, greatly exceeds actual rates of biosynthesis in vivo. Two 


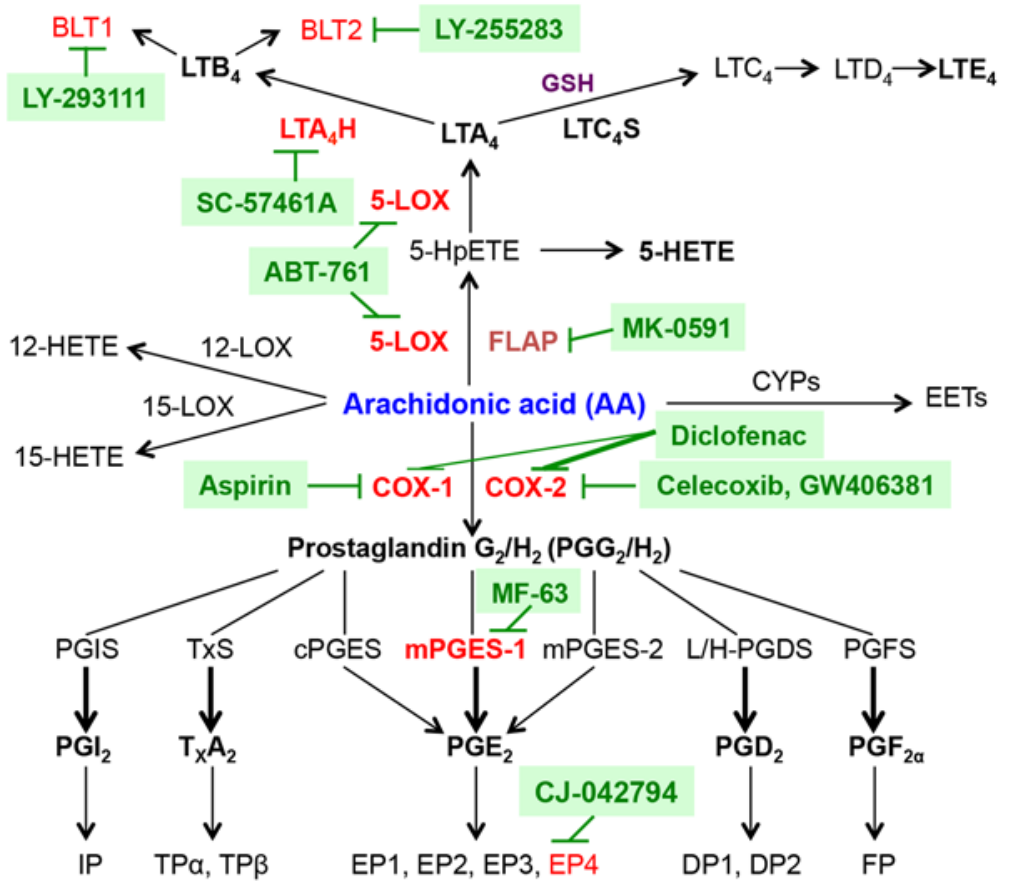

Figure 1. Antiinflammatory drug targets from the COX and 5-lipoxygenase pathways. Arachidonic acid (AA) is metabolized by 3 major enzymatic cascades: COX (COX-1/-2), lipoxygenase (5-LOX, 12-LOX, 15-LOX), and cytochrome P450 (CYP). The 2 COX isoforms, COX-1 and COX-2, oxidize AA to the hydroperoxy-endoperoxide PGG ${ }_{2}$ and then reduce $P G G_{2}$ to the unstable intermediate hydroxy-endoperoxide $\mathrm{PGH}_{2}$. $\mathrm{PGH}_{2}$ is transformed to eicosanoids $\mathrm{PGI}_{2}, \operatorname{TxA}_{2}, \mathrm{PGE}_{2}, \mathrm{PGD}_{2}$, and $\mathrm{PGF}_{2 \alpha}$ by the tissue-specific isomerases prostaglandin I synthase (PGIS), thromboxane synthase (TxS), prostaglandin E synthases (cPGES, mPGES-1, mPGES-2), prostaglandin D synthases (L/H-PGDS), and prostaglandin F synthase (PGFS), respectively. Each eicosanoid acts at the corresponding receptor distributed in various tissues. The 5-LOX enzyme, together with the accessory protein 5-LOX-activating protein (FLAP), catalyzes the conversion of AA to 5-hydroperoxyeicosatetraenoic acid (5-HpETE) and then to the unstable intermediate leukotriene $A_{4}\left(L_{T A}\right)$. 5-HpETE can be reduced by glutathione peroxidases into 5-HETE alcohol. The LTA 4 hydrolase $\left(\mathrm{LTA}_{4} \mathrm{H}\right)$ metabolizes LTA $_{4}$ to leukotriene $\mathrm{B}_{4}\left(\mathrm{LTB}_{4}\right)$, which acts at BLT1 and BLT2 receptors on target cells. Alternatively, LTA ${ }_{4}$ can be conjugated with glutathione (CSH) by $\mathrm{LTC}_{4}$ synthase ( $\mathrm{LTC}_{4} \mathrm{~S}$ ) to yield cysteinyl leukotrienes $\mathrm{LTC}_{4}, \mathrm{LTD}_{4}$, and $\mathrm{LTE}_{4}$, which act at CysLT receptors. Enzyme inhibitors and receptor antagonists are shown in green, while drug targets are depicted in red. CPGES, cytosolic PGE synthase; mPGES-1 and mPGES-2, microsomal PGE synthase- 1 and -2, respectively; L/H-PGDS, hematopoietic and lipocalin-type PGD synthases; IP, prostacyclin receptor; TP, thromboxane receptor; EP, E prostanoid receptor; DP, D prostanoid receptor; FP, F prostanoid receptor; EET, epoxyeicosatrienoic acid.

approaches to their analysis have informed drug development in this pathway. First, actual biosynthesis has been estimated by measurement (most accurately with mass spectrometry [MS]) of biologically inactive, but chemically stable, metabolites. The preferred target analyte is urine, as this avoids artefactual ex vivo cellular activation, is acquired noninvasively, and affords a time-integrated estimate of biosynthesis. A limitation of this approach is that the tissue and cellular origin of the measured metabolite is unknown, although this can be estimated indirectly in the case of the platelet (5). A second approach is to study, in vitro or ex vivo, the capacity of a defined cell type to generate a particular eicosanoid. The power of this approach is best illustrated by measurement of the inactive $\mathrm{Tx}$ hydrolysis product, $\mathrm{TxB}_{2}$, in stimulated platelet-rich plasma (6). This approach, particularly combined with measurement of urinary Tx metabolites, elucidated the pharmacology of low-dose aspirin, affording the basis for clinical trials that established its cardioprotective properties (2).

Here, we describe a MS-based capacity-related assay, applied in vitro or ex vivo to stimulated human whole blood but encompassing a broad swathe of the eicosanoid lipidome, not just a single compound. This permits us, in remarkably small sample sizes, to scan for the consequences of substrate rediversion after blockade of different enzymes or shifts in product formation consequent to receptor blockade in the eicosanoid biosynthetic/response pathways. We demonstrate unexpected consequences across the lipidome of inhibition of COXs and mPGES-1 and of receptor blockade and reveal how this approach can be 
A

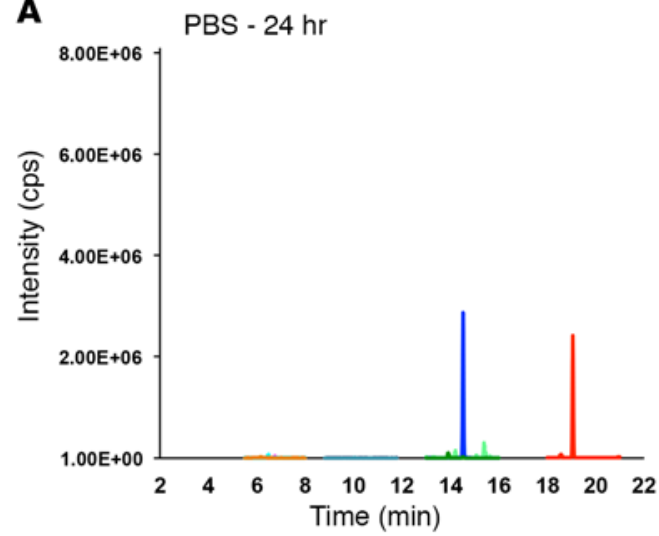

LPS - $24 \mathrm{hr}$

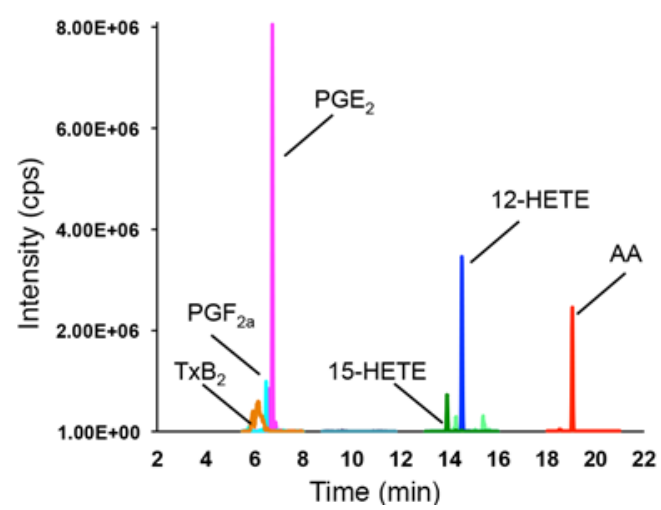

B

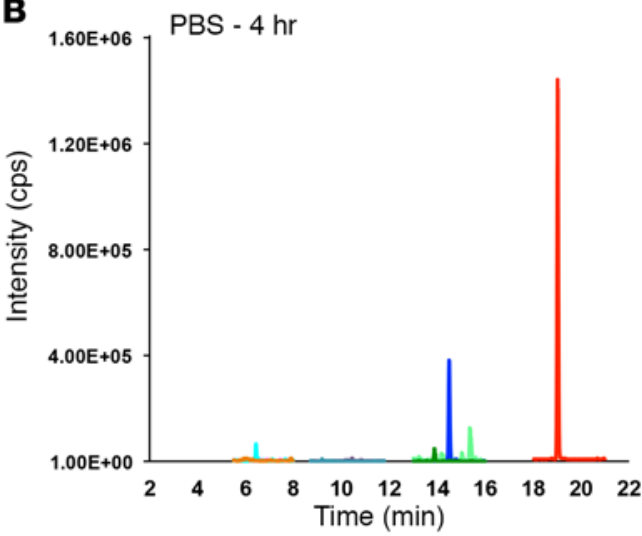

Zymosan $-4 \mathrm{hr}$

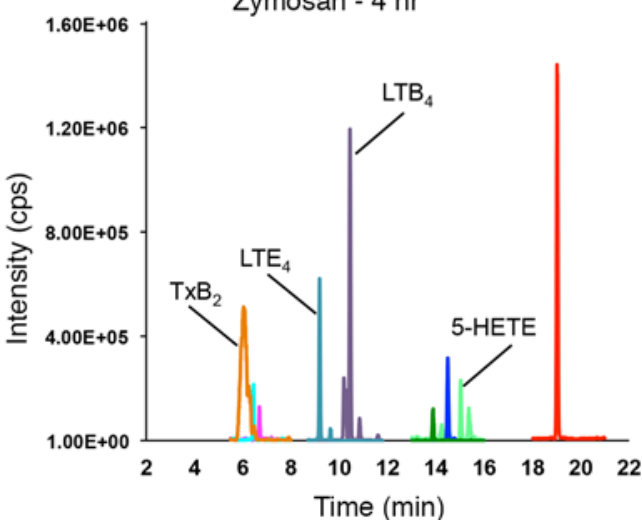

Figure 2. Ultra-performance liquid chromatography-tandem mass spectrometry profiling of bioactive eicosanoids in in vitro-stimulated human whole blood. Representative chromatograms of eicosanoids in whole blood stimulated with $100 \mu \mathrm{g} / \mathrm{ml}$ LPS for 24 hours (A) or activated with $125 \mu \mathrm{g} / \mathrm{ml}$ of zymosan (B) for 4 hours. Spectra of vehicle (PBS) controls at the corresponding time points are depicted in top panels. Selected peaks were identified by comparison with isotope-labeled standards. $\mathrm{PGE}_{2}$, prostaglandin $\mathrm{E}_{2 ;} \mathrm{PGF}_{2 a}$, prostaglandin $\mathrm{F}_{2 \mathrm{a}} ; \mathrm{TxB}_{2}$, thromboxane $\mathrm{B}_{2} ; \mathrm{LTB}_{4}$, leukotriene $\mathrm{B}_{4}$; LTE $_{4}$, leukotriene $\mathrm{E}_{4} ;$ 5-HETE, 5-hydroxyeicosatetraenoic acid; 12-HETE, 12-hydroxyeicosatetraenoic acid; 15-HETE, 15-hydroxyeicosatetraenoic acid; AA, arachidonic acid.

used to rationalize further evaluation of drug combinations and to scan for the biochemical consequences of combinatorial approaches to disruption of eicosanoid metabolism. Finally, using celecoxib as an example, we illustrate how the approach can be used to integrate preclinical and clinical studies by extrapolation of the in vitro results to whole blood ex vivo in patients dosed with the NSAID.

\section{Results}

Perturbations of the human plasma lipidome triggering COX and lipoxygenase product formation. Stimulation of whole blood with $100 \mu \mathrm{g} / \mathrm{ml} \mathrm{LPS}$ at $37^{\circ} \mathrm{C}$ demonstrated that PGs and Tx peaked at 24 hours and remained elevated throughout the rest of the time course of observation (Figure 2A, Figure 3B, and Supplemental Figure 1; supplemental material available online with this article; doi:10.1172/jci.insight.87031DS1). The production of $\mathrm{PGE}_{2}$ rose to $21.5 \pm 9.3 \mathrm{ng} / \mathrm{ml}$ (mean $\pm \mathrm{SD}, n=4, P=0.004$ ), while $\mathrm{PGF}_{2 \alpha}$ and $\mathrm{TxB}_{2}$ averaged $5.7 \pm 2.3$ $\mathrm{ng} / \mathrm{ml}(P=0.01)$ and $30.8 \pm 10.7 \mathrm{ng} / \mathrm{ml}(P=0.004)$, respectively (Supplemental Figure $1, \mathrm{~A}-\mathrm{C}$, and Supplemental Table 3$)$. In addition, LPS was a potent stimulus for formation of $15.3 \pm 2.3 \mathrm{ng} / \mathrm{ml}(P=0.01)$ total 15-hydroxyeicosatetraenoic acid (15-HETE) at 24 hours (Supplemental Figure 1E). Chiral analysis revealed that LPS induced both $(S)$ - and $(R)$-enantiomers of 15-HETE, which averaged $8.98 \pm 1.9 \mathrm{ng} / \mathrm{ml}(n=7, P=$ $0.04)$ and $4.6 \pm 1.2 \mathrm{ng} / \mathrm{ml}(P=0.03)$, respectively (Supplemental Figure 7, A and B). If 15-HETE production derived solely from the 15-lipoxygenase (15-LOX) enzyme, 15(S)-HETE would have been overwhelmingly the dominant product. These observations are consistent with its formation by COX enzymes $(7,8)$ or by autoxidation of eicosanoids (9). The production of total 12-HETE was time dependent and robust, resulting in a concentration of $1,292 \pm 190 \mathrm{ng} / \mathrm{ml}$ in activated blood by 48 hours (Supplemental Figure 1D); however, 
A
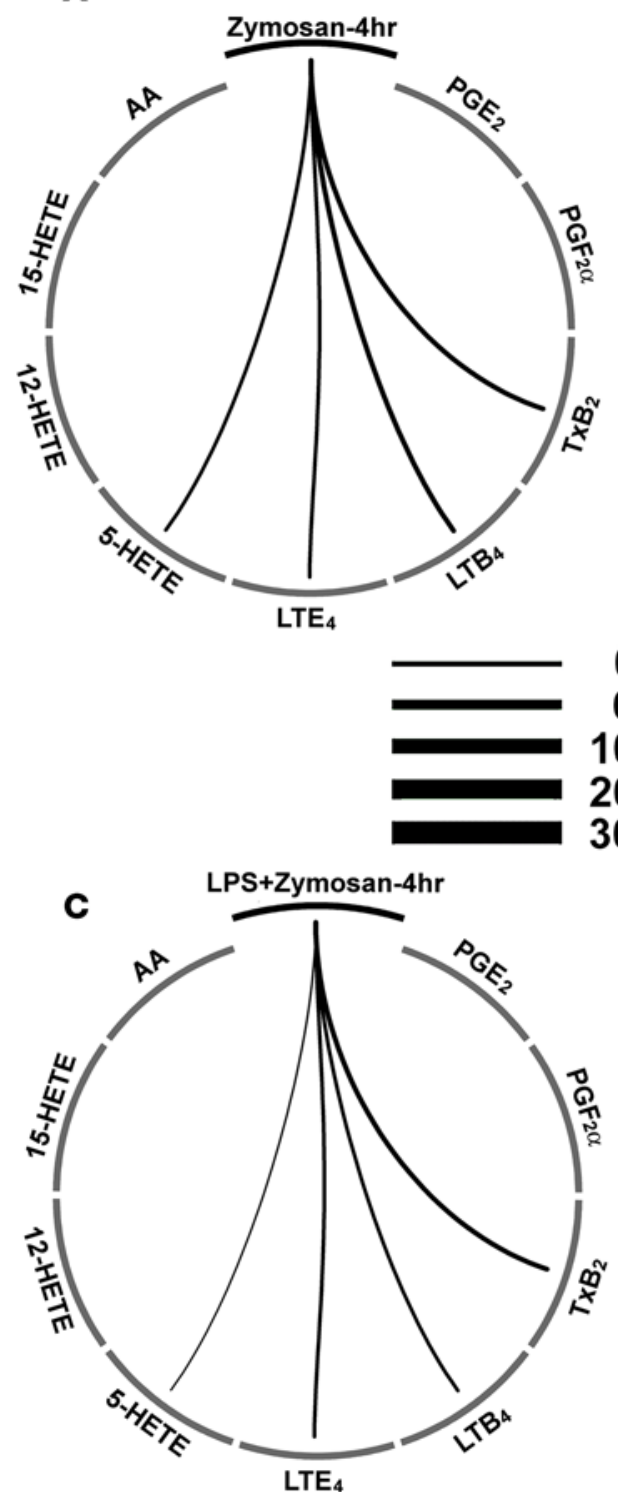

B

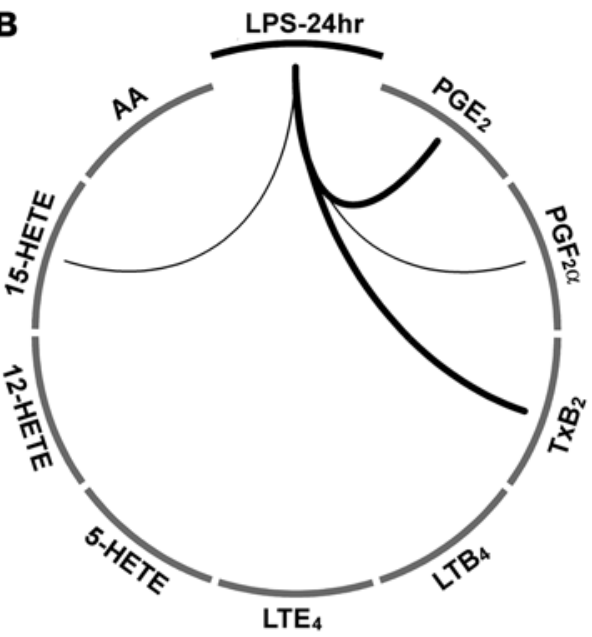

\section{$0 \leq \mathrm{ng} / \mathrm{ml}<6$ \\ $6 \leq \mathrm{ng} / \mathrm{ml}<10$ \\ $10 \leq \mathrm{ng} / \mathrm{ml}<20$ \\ $20 \leq \mathrm{ng} / \mathrm{ml}<30$ $30 \leq \mathrm{ng} / \mathrm{ml}<50$}

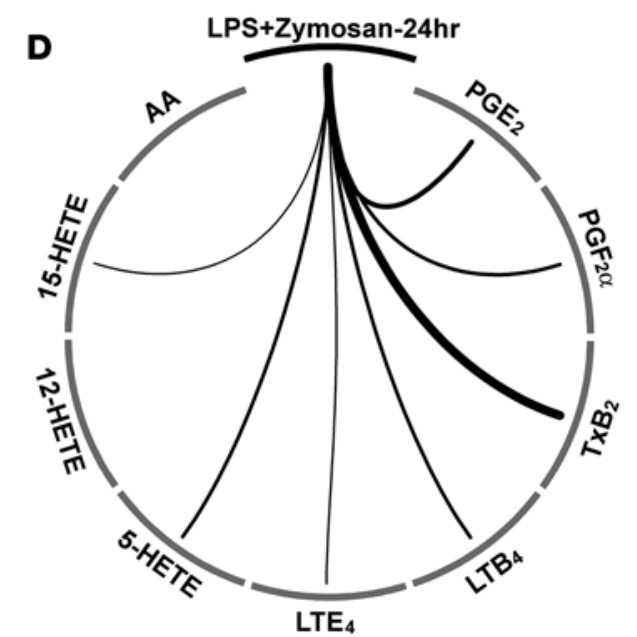

Figure 3. Human plasma eicosanoid production in response to activation of COX and/or lipoxygenase pathways in whole blood in vitro. Circos plots comparing plasma eicosanoid profiles of human whole blood stimulated with $100 \mu \mathrm{g} / \mathrm{ml}$ LPS or $125 \mu \mathrm{g} / \mathrm{ml}$ zymosan, alone or in combination, at 4 or 24 hours. Levels of lipids, significant ( $P<0.05$, unpaired, 2-tailed $t$ test) compared with PBS control at the corresponding time point, are shown after stimulation with zymosan for 4 hours $(\mathbf{A})(n=22)$, with LPS for 24 hours (B) $(n=22)$, with LPS and zymosan for 4 hours (C) $(n=16)$, and with LPS and zymosan for 24 hours (D) $(n=16)$. In vitro human whole-blood assay and ultra-performance liquid chromatography-tandem mass spectrometry (UPLC-MS/MS) analysis were performed as described in Methods. $P G E_{2}$, prostaglandin $E_{2} ; P_{2 F^{\prime}}$, prostaglandin $\mathrm{F}_{2 a^{\prime}} ; \mathrm{TxB}_{2}$, thromboxane $\mathrm{B}_{2}$; $\mathrm{LTB}_{4}$, leukotriene $\mathrm{B}_{4} ; \mathrm{LTE}_{4}$, leukotriene $\mathrm{E}_{4} ;$ 5-HETE, 5-hydroxyeicosatetraenoic acid; 12-HETE, 12-hydroxyeicosatetraenoic acid; 15-HETE, 15-hydroxyeicosatetraenoic acid; AA, arachidonic acid.

LPS stimulation did not affect 12-HETE levels, suggesting that a prolonged incubation of whole blood alone at $37^{\circ} \mathrm{C}$ resulted in spontaneous 12 -HETE release, most likely by activated platelets (10). Here, chiral analysis revealed that 12(S)-HETE was the predominant product, consistent with its formation by 12-LOX, and its formation was unaltered in human plasma by LPS (Supplemental Figure 8D). Finally, chiral analysis also revealed that LPS stimulated 11(R)-HETE production, whereas 11(S)-HETE, as well as both $(S)$ - and $(R)$-enantiomers of 8-HETE, were not detected in whole blood (Supplemental Figure 8A). Production of 5 -LOX-derived eicosanoids in the presence of LPS was at the lower limits of detection and was comparable to the vehicle control (data not shown). On the contrary, activation of whole blood with zymosan led to a time-dependent leukotriene (LT) and 5-HETE production, consistent with its effects on granulocytes, particularly neutrophil 5-LOX, in vivo $(11,12)$. Time-course and dose-response studies revealed that 125 $\mu \mathrm{g} / \mathrm{ml}$ zymosan induced a robust increase in 5-LOX products that peaked at 4 hours and averaged $8.43 \pm$ $4.12 \mathrm{ng} / \mathrm{ml}$ for $\mathrm{LTB}_{4}, 9.62 \pm 7.88 \mathrm{ng} / \mathrm{ml}$ for $\mathrm{LTE}_{4}$, and $15.1 \pm 6.37$ for 5 -HETE $(n=22)$ (Figure 2B, Figure 3A, Supplemental Figure 2, and Supplemental Table 3). Costimulation with LPS and zymosan for 4 hours resulted in a lipid profile with effects comparable to those from zymosan alone, suggesting that, at early times, eicosanoid release was driven by zymosan regardless of LPS. At 24 hours, coincubation with both stimuli led to a combined profile, consisting of lipids triggered by zymosan early on and eicosanoids whose biosynthesis depended on induction by LPS over a long-term incubation (Figure 3, C and D, and Supplemental Figure 4). 
A 4-hour incubation with the vehicle PBS resulted in detectable levels of $\mathrm{PGF}_{2 \alpha}(0.31 \pm 0.34 \mathrm{ng} / \mathrm{ml}$, mean \pm $\mathrm{SD}, n=22), \mathrm{TxB}_{2}(1.1 \pm 0.42 \mathrm{ng} / \mathrm{ml}), 5$-HETE $(1.43 \pm 0.6 \mathrm{ng} / \mathrm{ml}), 12-\mathrm{HETE}(21.7 \pm 20.6 \mathrm{ng} / \mathrm{ml}), 15-\mathrm{HETE}$ $(0.5 \pm 0.2 \mathrm{ng} / \mathrm{ml})$, and AA $(2,613 \pm 1,327 \mathrm{ng} / \mathrm{ml})$. By 24 hours, most lipids were unaltered, but 12-HETE and AA increased 6-fold (141 $\pm 115 \mathrm{ng} / \mathrm{ml})$ and 2-fold (5,216 \pm 2,140 $\mathrm{ng} / \mathrm{ml})$, respectively (Supplemental Figure 3 , A and B), consistent with continuous secretion of 12-HETE by platelets and a dynamic release of AA by all blood cell types. $\mathrm{PGD}_{2}$ and $\mathrm{PGI}_{2}$ production was not detected in human plasma. Amounts of 20-HETE did not differ between stimulated or vehicle-treated whole blood (Supplemental Figure 1F). Low (pg/ml) levels of 12-hydroxy-5Z,8E,10E-heptadecatrienoic acid (12-HHT) were detected in plasma in response to LPS or zymosan, while a more robust production was detected in sera $(12.38 \pm 1.41 \mathrm{ng} / \mathrm{ml}$, mean $\pm \mathrm{SD}, n=4)$, and inhibited by diclofenac, consistent with the origin of 12-HHT from the platelet-derived COX-1/thromboxane synthase (COX-1/TxS) axis (Supplemental Figure 5, C and D). The "specialized proresolving mediators," resolvins $\mathrm{D} 1(\mathrm{RvD} 1)$ and $\mathrm{D} 2(\mathrm{RvD} 2)$, maresin 1, and protectin, were not detected in this study. Lipoxin $\mathrm{A}_{4}$ $\left(\mathrm{LXA}_{4}\right.$ ) was not detected at baseline but could be induced by zymosan at 24 hours; while modest levels of resolvin E1 (RvE1) were measured in untreated plasma, it was not induced by either stimulus (Supplemental Figure 5, A and B). The fatty acids eicosapentaenoic acid (EPA), docosahexaenoic acid (DHA), and AA were not significantly affected by whole blood stimulation (data not shown). Thus, for the purposes of increased LC-MS efficiency, incubation with authentic standards was reduced from 21 to 9 analytes $\left(\mathrm{PGE}_{2}, \mathrm{PGF}_{2 \alpha}\right.$, $\mathrm{TxB}_{2}, \mathrm{LTE}_{4}, \mathrm{LTB}_{4}, 5$-HETE, 12-HETE, 15-HETE, and AA). In summary, a 24-hour incubation of human whole blood with LPS $(100 \mu \mathrm{g} / \mathrm{ml})$ maximized activation of the COX-1/-2 pathways, while incubation with zymosan $(125 \mu \mathrm{g} / \mathrm{ml})$ for 4 hours yielded maximum activation of the 5-LOX pathway.

Substrate rediversion consequent to inhibition of $m P G E S-1$. To determine how pharmacological blockade of specific targets in the COX and LOX pathways affects the human plasma lipidome in vitro, we screened the selective COX-2 inhibitors (COX-2i), celecoxib and GW406381; the COX-1/-2 inhibitor (COX-1/-2i), diclofenac; aspirin (at concentrations corresponding to low-dose ( $<100 \mathrm{mg} / \mathrm{d}$ ) administration in vivo, which would preferentially target COX-1)); and the E prostanoid receptor 4 (EP4) antagonist, CJ-042794, and compared their effects on the lipidome to those of the mPGES-1 inhibitor (mPGES-1i), MF-63 (Figure 4). We observed large interindividual variability in the capacity to form eicosanoids among donors in each treatment group (Supplemental Tables 4 and 5) that constrained comparison of absolute eicosanoid concentrations in studies of small sample size. However, expression of drug effects as a percentage of their own stimulus control for each subject addressed this limitation by reducing variance. Inhibition of mPGES-1 in whole blood by MF-63 at its $\mathrm{IC}_{50}$ concentration of $0.8 \mu \mathrm{M}$ or by $10 \mu \mathrm{M}$ had no significant effect on LPS-triggered $\mathrm{PGE}_{2}$ because of variance if the data were expressed in absolute values. However, when the same data were expressed as a percentage of stimulus control, a $60 \%$ reduction in $\mathrm{PGE}_{2}$ relative to the untreated, stimulated control ( $n=5, P=0.003$ ) was observed with $10 \mu \mathrm{M}$ MF-63. Concurrently, there was an elevation in PGF (by an average $50 \%, P=0.008$ ) and $\mathrm{TxB}_{2}$ (by $37 \%, P=0.007$ ), consistent with rediversion of the $\mathrm{PGH}_{2}$ substrate of mPGES-1 to PGF synthase (PGFS) and TxS, respectively. Although total 15-HETE was apparently elevated by approximately $10 \%$ on average $(P=0.048)$, this was not confirmed by chiral analysis (vide infra). The marginal effect of mPGES-1 inhibition on 15-HETE was in contrast to the effect of COX-1/-2 blockade. At $10 \mu \mathrm{M}$, the COX-2i, celecoxib $(n=5)$ and GW406381 $(n=5)$, and the COX-1/-2i, diclofenac $(n$ $=4$ ), profoundly reduced $\mathrm{PGE}_{2}$ and $\mathrm{PGF}_{2 \alpha}$ (both by more than $90 \%, P<0.0001$ ), $\mathrm{TxB}_{2}$ (by more than $80 \%$, $P<0.0001$ ), and total 15-HETE (by more than 70\%, $P<0.001$ ) (Figure $4, \mathrm{~A}-\mathrm{C}$ ). Chiral analysis confirmed the inhibitory effect of celecoxib on in vitro plasma levels of 15(S)-HETE and 15(R)-HETE, rather than solely the 15-LOX product, 15(S)-HETE. Suppression of 11(R)-HETE, but not the platelet 12-LOX product, 12(S)-HETE, was observed with celecoxib in vitro, in contrast to the mPGES-1i, MF-63, that had no effect on 11(R)-HETE (Supplemental Figure 8B). Neither 15(R)- nor 15(S)-HETE were suppressed by MF-63. Celecoxib also inhibited $\mathrm{LTB}_{4}$ by $40 \%(P=0.02)$ and $\mathrm{LTE}_{4}$ by $30 \%(P=0.043)$ but had no effect on 5-HETE levels, suggesting that LT inhibition happened downstream of the 5-LOX enzyme. Similarly to COX-2i, aspirin $(n=4)$ inhibited $\mathrm{PGF}_{2 a}$ (by 70\%, $P<0.0001$ ), $\mathrm{TxB}_{2}$ (by 65\%, $P<0.0001$ ), and total 15-HETE (by $35 \%, P=0.0003$ ), but unlike the COX-2i, the low concentration of aspirin had no effect on $\mathrm{PGE}_{2}$, suggesting its predominant formation from COX-2 in this setting (Figure 4D). Interestingly, aspirin potentiated total 12-HETE by $30 \%(P=0.01)$, consistent with inhibition of platelet COX-1, making more substrate available for the platelet 12-LOX enzyme. Finally, the EP4 antagonist $(n=5)$ did not affect the plasma lipidome, suggesting that such a pharmacological perturbation did not result in feedback mechanisms to compensate for the lack of signaling through EP4 (data not shown). At their $\mathrm{IC}_{50}$ s, MF-63 reduced $\mathrm{PGE}_{2}$ and elevated 

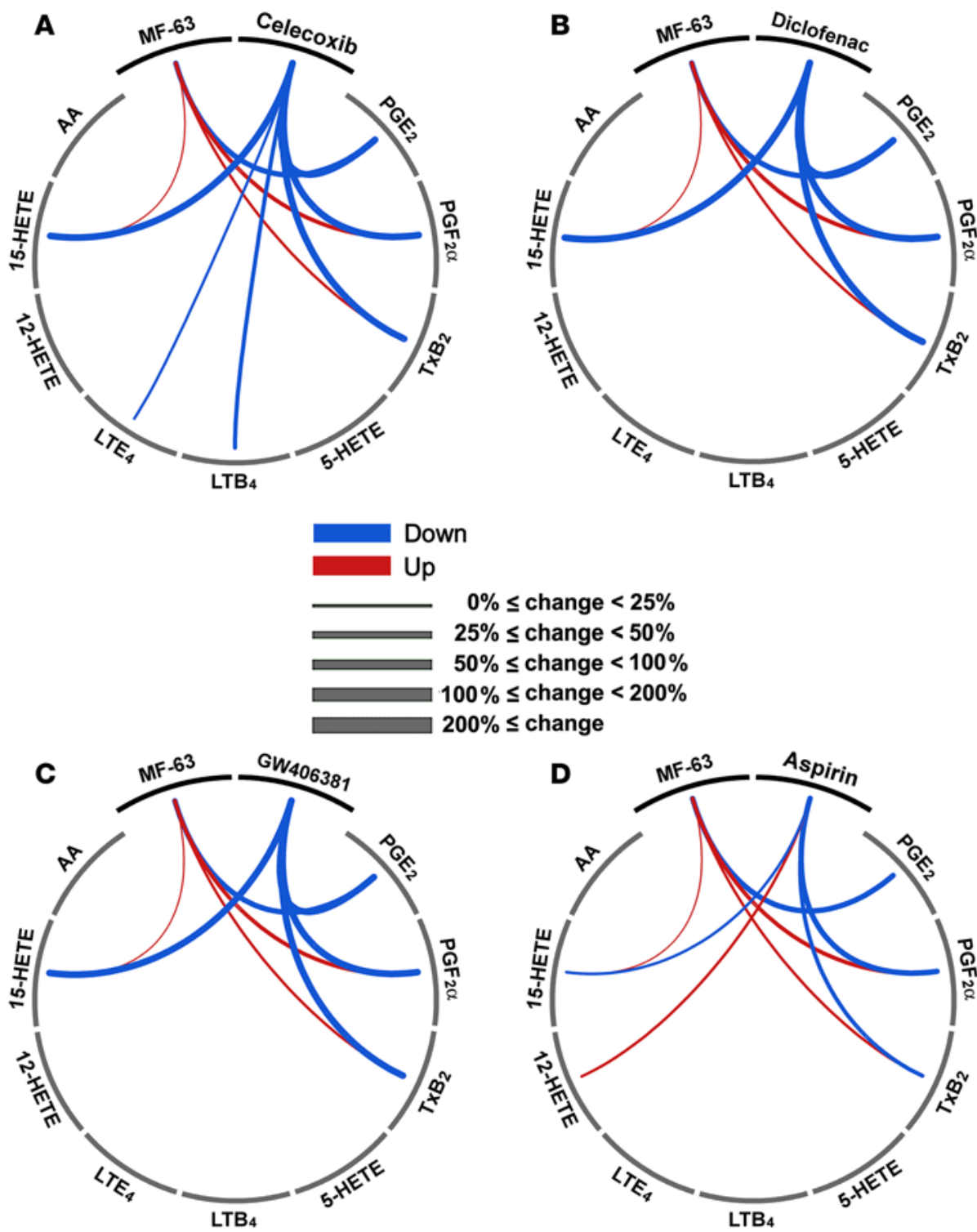

Down
$0 \% \leq$ change $<25 \%$

$25 \% \leq$ change $<50 \%$

$50 \% \leq$ change $<100 \%$ $100 \% \leq$ change $<200 \%$ $200 \% \leq$ change

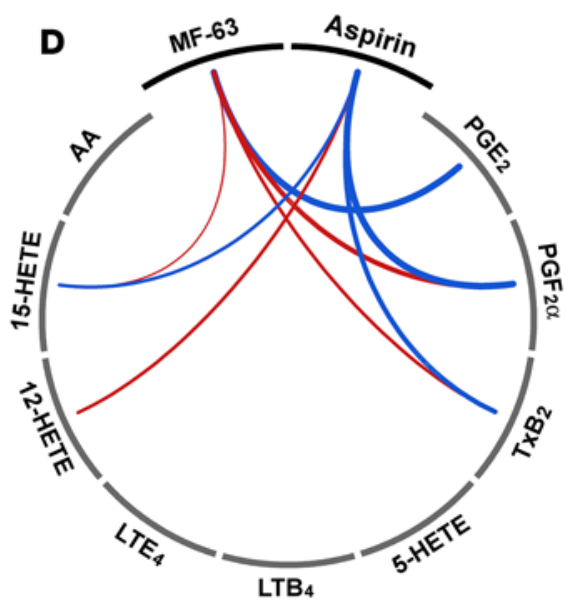

Figure 4. Pharmacological inhibition of microsomal prostaglandin E synthase- 1 exerts effects on human plasma lipidome in vitro that are distinct from COX-1/-2 blockade. Circos plots comparing plasma eicosanoid profiles of human whole blood stimulated with $100 \mu \mathrm{g} / \mathrm{ml}$ LPS and treated with the microsomal prostaglandin E synthase-1 (mPGES-1) inhibitor, MF-63, $(n=5)$ or treated with COX inhibitors celecoxib (A) $(n=5)$, diclofenac (B) $(n=5)$, GW406381 (C) $(n=5)$, or aspirin (D) $(n=4)$. All compounds, except aspirin (1.5 mM), were used at a concentration of $10 \mu \mathrm{M}$. In vitro human wholeblood assay and ultra-performance liquid chromatography-tandem mass spectrometry (UPLC-MS/MS) analysis were performed as described in Methods. Data are expressed as percentage of LPS+DMSO control. Red lines indicate significantly $(P<0.05$, unpaired, 2 -tailed $t$ test) elevated levels of the corresponding lipid, while blue lines indicate significant $(P<0.05$, unpaired, 2 -tailed $t$ test) reductions; the thickness of lines represents the degree of change. $P G E_{2}$, prostaglandin $E_{2}$; $\mathrm{PGF}_{2 \alpha^{\prime}}$, prostaglandin $\mathrm{F}_{2 \alpha} ; \mathrm{TxB}_{2}$, thromboxane $\mathrm{B}_{2} ; \mathrm{LTB}_{4}$, leukotriene $\mathrm{B}_{4} ; \mathrm{LTE}_{4}$, leukotriene $\mathrm{E}_{4} ; 5$-HETE, 5-hydroxyeicosatetraenoic acid; 12-HETE, 12-hydroxyeicosatetraenoic acid; 15-HETE, 15-hydroxyeicosatetraenoic acid; AA, arachidonic acid.

$\mathrm{TxB}_{2}$, while celecoxib and diclofenac reduced $\mathrm{PGE}_{2}, \mathrm{PGF}_{2 \alpha}, \mathrm{TxB}_{2}$, and total 15-HETE (Supplemental Figure 6 and Supplemental Table 5). Similar trends were observed after targeted inhibition of the 5-LOX pathway (Supplemental Figure 9). Inhibition of $\mathrm{LTA}_{4}$ hydrolase $\left(\mathrm{LTA}_{4} \mathrm{H}\right)$ resulted in a dose-dependent reduction in $\mathrm{LTB}_{4}$ (by $25 \%, P=0.05$, and $90 \%, P=0.0001$, at $\mathrm{IC}_{50}$ and $10 \mu \mathrm{M}$, respectively, $n=5$ ), with a concurrent elevation in $\mathrm{LTE}_{4}$ levels (by $38 \%, P=0.02$, and $170 \%, P=0.01$, at $\mathrm{IC}_{50}$ and $10 \mu \mathrm{M}$, respectively, $n=4$ ). Such an effect is consistent with shunting of the common substrate $\mathrm{LTA}_{4}$ from hydrolysis to $\mathrm{LTB}_{4}$ toward the enzyme $\mathrm{LTC}_{4}$ synthase ( $\mathrm{LTC}_{4} \mathrm{~S}$ ), which converts $\mathrm{LTA}_{4}$ and glutathione to $\mathrm{LTC}_{4}$ and then subsequently forms $\mathrm{LTE}_{4}$. As expected, inhibition of both 5-LOX and 5-LOX-activating protein (FLAP) reduced levels of $\mathrm{LTB}_{4}, \mathrm{LTE}_{4}$, and 5-HETE. Interestingly, antagonism of the BLT2 $(n=5)$, but not the BLT1 $(n=5)$, receptor for $\mathrm{LTB}_{4}$ elevated levels of 5-HETE (by $20 \%, P=0.02$ ) and $\mathrm{LTE}_{4}$ (by $22 \%, P=0.0009$ ) at their $\mathrm{IC}_{50}$ s, but not at the fixed concentration of $10 \mu \mathrm{M}$ (Supplemental Figure 9).

Treatment of whole blood with the vehicle DMSO for 4 hours led to detectable levels of total 5-HETE $(0.7 \pm 0.1 \mathrm{ng} / \mathrm{ml}), 12$-HETE $(13.5 \pm 12 \mathrm{ng} / \mathrm{ml}), 15$-HETE $(0.4 \pm 0.1 \mathrm{ng} / \mathrm{ml})$, and AA $(1,405 \pm 238 \mathrm{ng} /$ $\mathrm{ml})$. Similarly to PBS, prolongation of incubation to 24 hours did not further alter 5-HETE and 15-HETE, while 12-HETE and AA increased 10-fold (134 $\pm 85 \mathrm{ng} / \mathrm{ml})$ and 2-fold $(2,724 \pm 84 \mathrm{ng} / \mathrm{ml})$, respectively (Supplemental Figure 3, C and D). DMSO constituted $0.4 \%$ of total sample volume and did not trigger hemolysis or otherwise visible cell toxicity. 
A

$4 \mathrm{hr}$

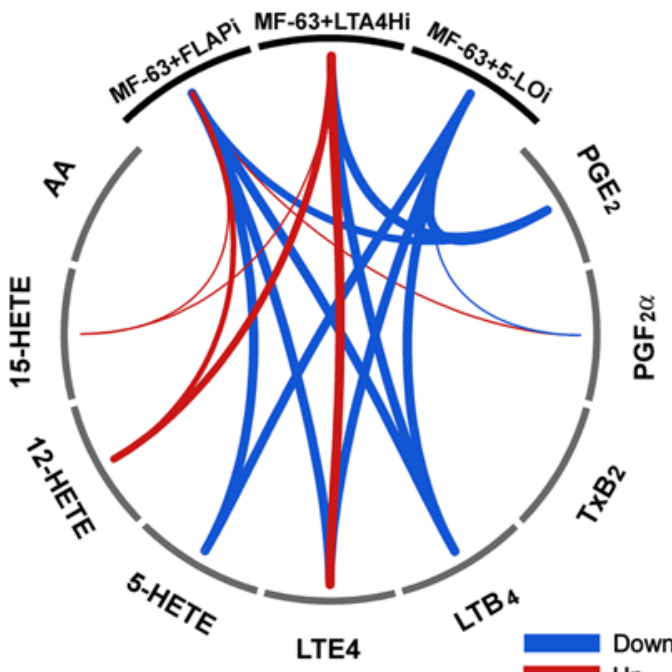

B
$24 \mathrm{hr}$

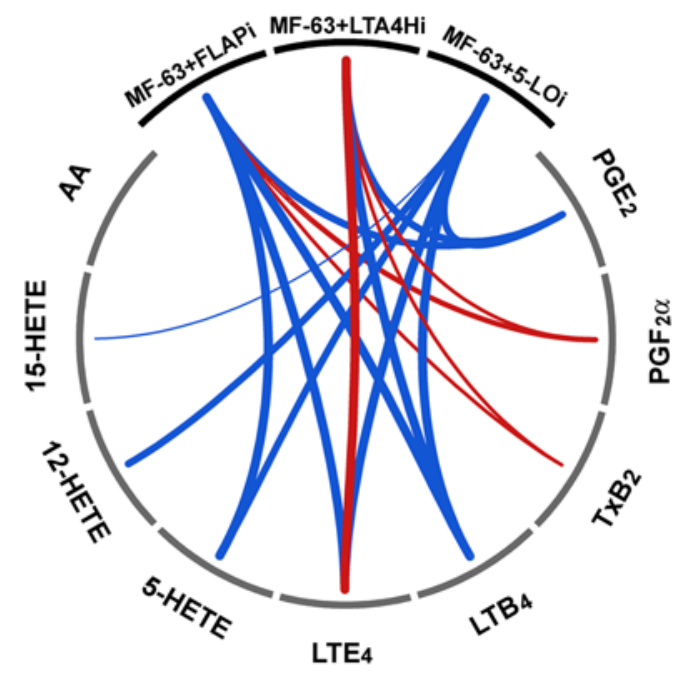

Up

$0 \% \leq$ change $<25 \%$

$25 \% \leq$ change $<50 \%$

$50 \% \leq$ change $<100 \%$

$100 \% \leq$ change $<200 \%$

$200 \% \leq$ change

Figure 5. Combinations of microsomal prostaglandin E synthase-1 inhibitor with compounds targeting distinctly the 5-lipoxygenase cascade have variable effects on the human plasma lipidome in vitro. Human whole blood was stimulated with $100 \mu \mathrm{g} / \mathrm{ml}$ LPS and $125 \mu \mathrm{g} / \mathrm{ml}$ zymosan and treated with the microsomal prostaglandin $\mathrm{E}$ synthase-1 (mPGES-1) inhibitor, MF-63, in combination with the 5-lipoxygenase-activating (5-LOX-activating) protein (FLAP) inhibitor, MK-0591, or with the leukotriene $A_{4}$ hydrolase (LTA $H$ ) inhibitor, SC-57461A, or with the 5-LOX inhibitor, ABT-761, for 4 hours (A) or 24 hours (B). All compounds were used at $10 \mu \mathrm{M}$ concentration. In vitro human whole-blood assay and ultra-performance liquid chromatography-tandem mass spectrometry (UPLC-MS/ MS) analysis were performed as described in Methods. Data are expressed as percentage of LPS+zymosan+DMSO control. Red lines indicate significantly $(P<0.05, n=5$, unpaired, 2-tailed $t$ test) elevated levels of the corresponding lipid, while blue lines indicate significant $(P<0.05, \mathrm{n}=5$, unpaired, 2 -tailed $t$ test) reductions; thickness of lines

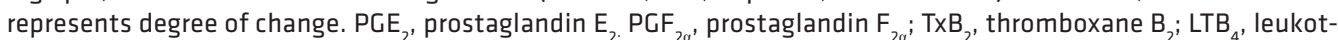
riene $\mathrm{B}_{4} ; \mathrm{LTE}_{4}$, leukotriene $\mathrm{E}_{4}$; 5-HETE, 5-hydroxyeicosatetraenoic acid; 12-HETE, 12-hydroxyeicosatetraenoic acid; 15-HETE, 15-hydroxyeicosatetraenoic acid; AA, arachidonic acid.

Overall, despite only 4 to 5 donors in each experiment, the approach captured dose-dependent effects of the studied compounds, demonstrated substrate shunting within and between both COX and LOX pathways, and segregated the lipidomic profiles consequent to whole-blood perturbation with COX-1/-2 versus mPGES-1 inhibitors.

Screening of combinatorial approaches to antiinflammatory drug therapy. Considering that products of AA metabolism from both COX and LOX pathways may contribute to inflammation $(13,14)$, dual inhibitors of 5-LOX and COX cascades have been investigated (15). Here, we tested how coincubations of a COX-2i, an mPGES-1i, or an EP4 antagonist with inhibitors of specific components of the 5-LOX cascade (5-LOX, FLAP, or $\mathrm{LTA}_{4} \mathrm{H}$ ) would affect the plasma lipidome. Results for combinations with the mPGES-1i demonstrate common features of this approach (Figure 5). Time-dependent effects on plasma lipidome were clearly evident. A 4-hour coincubation of the mPGES-1i with the FLAP inhibitor (FLAPi) $(n=5)$ resulted in almost complete abrogation of LT formation $(P<0.0001)$ and an $80 \%$ reduction in 5-HETE levels $(P<$ 0.0001 ), consistent with inhibition of the FLAP, while total 12-HETE and 15-HETE were elevated by $85 \%$ $(P=0.02)$ and $20 \%(P=0.006)$, respectively (Figure $5 \mathrm{~A})$. However, these elevations were only transient and undetected by 24 hours (Figure 5B). At 24 hours, the inhibitory effect on 5-LOX products was sustained and $\mathrm{PGE}_{2}$ was reduced on average by $52 \%(P<0.0001)$, while $\mathrm{PGF}_{2 \alpha}$ and $\mathrm{TxB}_{2}$ were increased by $53 \%(P=$ $0.002)$ and $28 \%(P=0.0004)$, respectively. The latter effects on PGs and Tx are characteristic of mPGES-1 inhibition by MF-63 (Figure 4). In contrast to combination with the FLAPi, coincubation of MF-63 with the 5-LOXi ABT-761 did not result in elevation of plasma lipids but rather had profound inhibitory effects on various eicosanoids. A 4-hour cotreatment with the 5-LOXi resulted in a reduction in LTs (by $95 \%, P<$ 

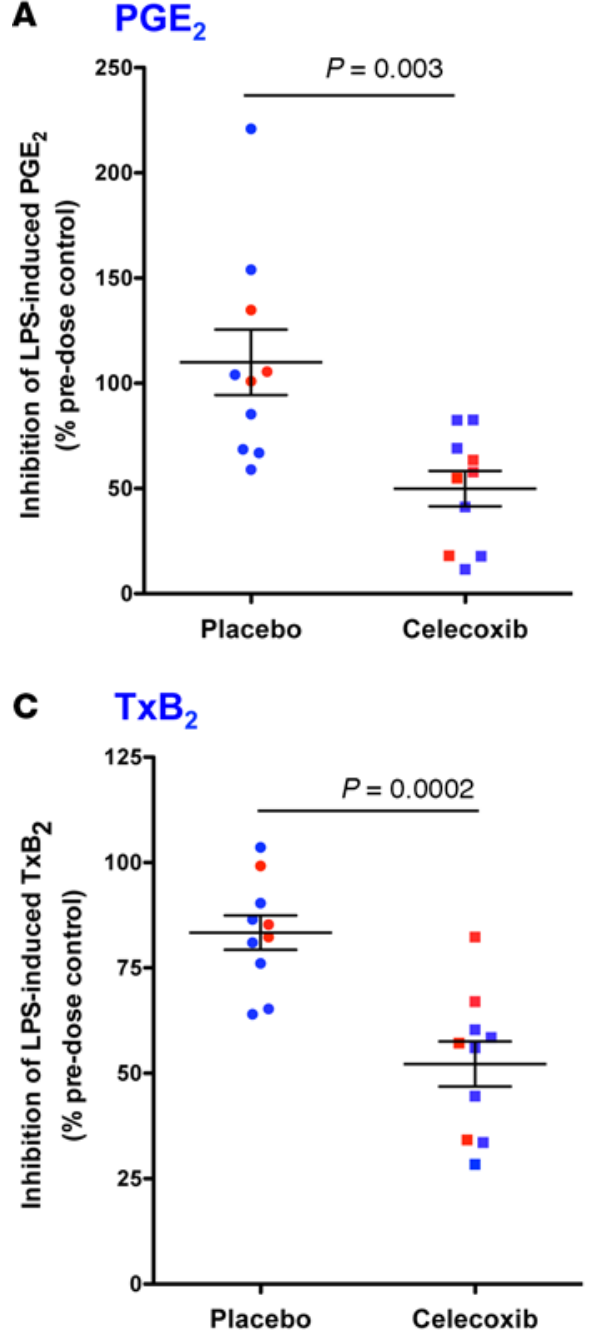

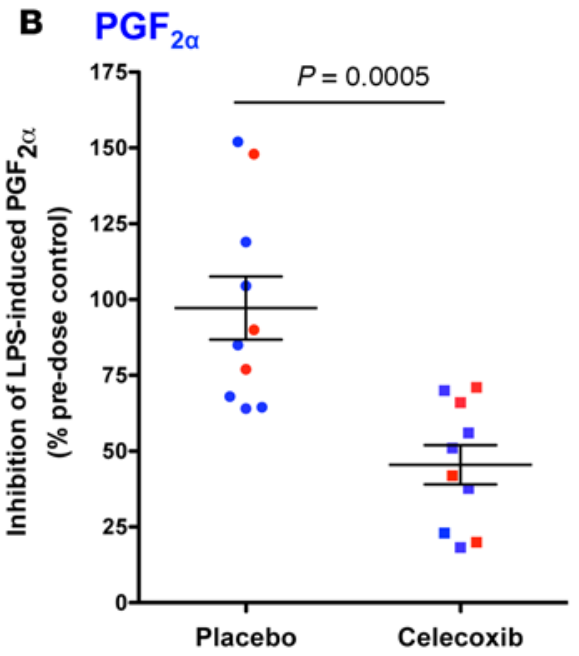

D 15-HETE

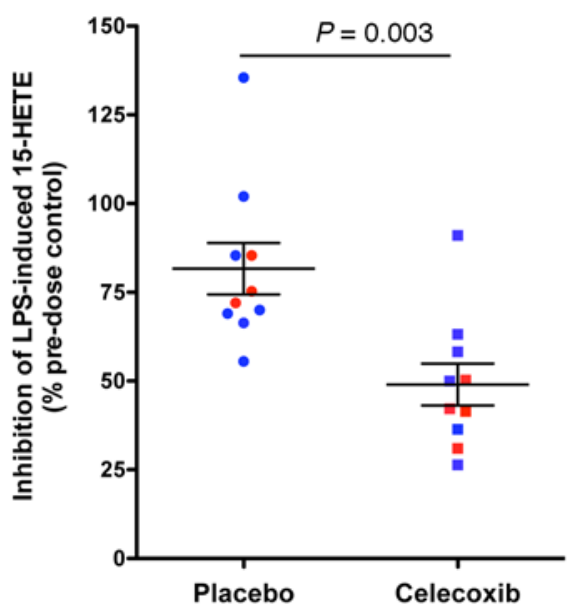

Figure 6. Ex vivo effects of the COX-2 inhibitor, celecoxib, on plasma lipidome in healthy subjects. Whole blood was collected before (pre-dose) and 3 hours after (post-dose) celecoxib or placebo administration. Plasma levels of prostaglandin $E_{2}\left(P G E_{2}\right)(A)$, prostaglandin $F_{2 a}$ $\left(\mathrm{PGF}_{2 \alpha}\right)(\mathbf{B})$, thromboxane $\mathrm{B}_{2}\left(\mathrm{TxB}_{2}\right)(\mathbf{C})$, and 15-hydroxyeicosatetraenoic acid (15-HETE) (D) expressed as percentage of pre-dose control. Ex vivo human whole blood assay and ultra-performance liquid chromatography-tandem mass spectrometry (UPLC-MS/MS) analysis were performed as described in Methods. Red and blue dots represent female and male subjects, respectively. Data represent mean \pm SEM. Unpaired, 2-tailed $t$ test, $n=10 /$ group.

0.0001), 5-HETE (by 76\% $(P<0.0001)$, and $\mathrm{PGE}_{2}$ (by $60 \%, P<0.0001$ ) and a mild and transient decrease in $\mathrm{PGF}_{2 \alpha}$ (by $14 \%, P=0.004$ ). Total 12 -HETE and 15-HETE were also inhibited on average by $54 \%(P=0.002)$ and $16 \%(P$ $=0.02)$ at 24 hours, accordingly, which might be due to nonspecific effects of the 5-LOXi on 12-LOX and on COX-2 or 15-LOX after a prolonged incubation. Chiral analysis to make the latter distinction was not performed. Surprisingly, with this drug combination, we did not observe the elevation in $\mathrm{PGF}_{2 a}$ and $\mathrm{TxB}_{2}$ observed with mPGES-1 inhibition by MF-63 alone (Figure 4).

Coincubation of MF-63 with the LTA $_{4}$ Hi SC-57461A for 4 or 24 hours reduced LTB $_{4}$ by $90 \%(P<0.0001)$ but elevated LTE $_{4}$ by more than $200 \%(P=0.0006)$ consistent with the $\mathrm{LTA}_{4} \mathrm{H}$ inhibition observed in a single-compound screen (Supplemental Figure 9). Similar to the combination with the FLAP, short-term coincubation of MF-63 with the $\mathrm{LTA}_{4} \mathrm{H}$ inhibitor $\left(\mathrm{LTA}_{4} \mathrm{Hi}\right)(n=5)$ led to transient increases of total 12 -HETE (by $52 \%, P=0.03$ ) and 15-HETE (by $16 \%, P=0.02$ ) that had dissipated by 24 hours, by which time $\mathrm{PGF}_{2 \alpha}$ and $\mathrm{TxB}_{2}$ had increased by $44 \%(P=0.03)$ and $38 \%(P=0.015)$, respectively. $\mathrm{PGE}_{2}$ was reduced throughout the whole experiment by more than $50 \%(P=0.001)$ (Figure 5$)$.

The effect of drug combinations on plasma lipidome was stimulus related (Supplemental Figure 10), consistent with different blood cell types that are activated and engaged in the response to drug inhibition.

Validation of the in vitro whole-blood assay in an ex vivo setting. We validated our in vitro assay by its application ex vivo after administration of celecoxib to healthy volunteers. A single oral dose led to a reduction in the ex vivo LPS-stimulated plasma levels of $\mathrm{PGE}_{2}(49.9 \% \pm 27 \%$ of pre-dose control, mean $\pm \mathrm{SD}, P=$ $0.003, n=10), \mathrm{PGF}_{2 \alpha}(45.5 \% \pm 20.5 \%, P=0.0005), \mathrm{TxB}_{2}(52.2 \% \pm 16.9 \%, P=0.0002)$, and total 15-HETE $(49 \% \pm 18.7 \%, P=0.003)$ (Figure 6). These findings are consistent with the in vitro celecoxib results (Figure 4). Chiral analysis of the ex vivo samples showed that celecoxib tended to inhibit both 15(S)-HETE and 15(R)-HETE, although the data reached significance ex vivo only with the ( $S$ )-enantiomer (Supplemental Figure 7). Here, despite suppression of $11(R)$-HETE by celecoxib in vitro, it was not suppressed by celecoxib ex vivo (Supplemental Figure 8). Urinary metabolites (M), PGI-M and PGE-M, indices of systemic $\mathrm{PGI}_{2}$ and $\mathrm{PGE}_{2}$ biosynthesis, respectively, were reduced in the celecoxib-treated group (Supplemental Figure 11). However, urinary Tx-M was comparable to placebo control, indicating that although the capacity of blood cells to generate Tx in vitro and ex vivo was modestly reduced, actual biosynthesis of Tx was not 
significantly altered by administration of a COX-2i as previously observed (16). Indeed, consistent with an effect on platelet capacity, we have previously reported that celecoxib inhibits serum $\mathrm{TxB}_{2}$ ex vivo but to a degree insufficient to inhibit platelet aggregation (17). Urinary metabolites of $\mathrm{PGD}_{2}$ and the isoprostane, $\mathrm{iPF}_{2 \alpha}-\mathrm{VI}$, were not affected by celecoxib treatment (data not shown).

\section{Discussion}

A broad-spectrum, targeted lipidomics approach based on ultra-performance liquid chromatography-tandem MS (UPLC-MS/MS) is reported. It is a rapid, sensitive, analytical method that allows simultaneous profiling and high-throughput, quantitative analysis of COX and LOX products in human whole blood in vitro and ex vivo. Using small sample sizes, we can reproducibly screen compounds targeting specific eicosanoid biosynthetic enzymes or receptors for their dose-dependent effects, mechanisms of action, and combinatorial potential. The method was validated by extrapolating in vitro data to ex vivo results after in vivo administration of a COX-2i to volunteers. Thus, the method can be applied to proof-of-concept experiments in drug development and can be used to integrate preclinical and clinical studies.

Differential perturbations of the lipidome with LPS and zymosan allow for scanning a wide range of diverse products of the COX and LOX pathways. The whole-blood model provides near physiological conditions in a plasma protein-rich environment and in the presence of blood cells that participate in transcellular biosynthesis of eicosanoids (18). Stimulus-dependent effects of drug combinations on the plasma lipidome vividly depict how activation of different blood cell types affects the response to drug inhibition. The capacity of cells to generate a particular class of eicosanoids is limited by their ability to respond to a particular agonist and the availability of the relevant enzymatic machinery to engage in single-cell or transcellular lipid biosynthesis $(18,19)$. Human peripheral blood monocytes express both COX isoforms and provide a major source of eicosanoids in capacity-related assays in plasma $(20,21)$. Monocyte-derived $\mathrm{TxB}_{2}$ and $\mathrm{PGE}_{2}$ largely depend on the induction of COX-2 by exogenous agonists, such as LPS (20, 21). Polymorphonuclear neutrophils respond to LPS with induction of COX-2 (22-24). Zymosan also induces neutrophil COX-2 expression and production of $\mathrm{TxB}_{2}$ and $\mathrm{PGE}_{2}$ (25). Platelets express COX-1 and 12-LOX and are the major source of $\mathrm{TxA}_{2}$ and 12-HETE in serum. Besides TxA 2 , platelet TxS catalyzes production of 12-HHT, a potential ligand for the $\operatorname{LTB}_{4}$ receptor BLT2 $(26,27)$.

Although this high-throughput assay quantitates precisely a range of lipids, it is not designed to attribute them to their cellular origins in a complex matrix, such as whole human blood. Nevertheless the combination of drug intervention and chiral analysis allows for some tentative conclusions. Suppression of induced levels of TxB2 and 12-HHT and elevation of 12- HETE by aspirin at concentrations corresponding to peak plasma levels after administration of low doses, points to these compounds being products of the COX-1 pathway in platelets. While induced total 15-HETE was suppressed by COX-2i, this applied to both enantiomers, not just 15(S)-HETE, the product of 15-LOX, which is expressed in monocytes and eosinophils (28-30). 15(R)-HETE is increased by LPS with kinetics that correspond to COX-2 induction and is suppressed, along with 11(R)-HETE, by celecoxib, consistent with its formation by COX-2. 15(S)-HETE may also be formed by COX-2 (7). However, while the nonenzymatic products 8(R)-HETE and 8(S)-HETE were not detected in human plasma, the possibility that some of 15(S)-HETE was made via this route cannot be excluded. A more likely explanation is that it may be the product of eosinophil 15-LOX, activated by formation of COX-2-derived eicosanoids in monocytes (31). Celecoxib, diclofenac, and the COX-2i GW406381 may thus suppress both 15-HETE enantiomers in stimulated whole blood by directly inhibiting COX-2, indirectly suppressing eosinophil 15-LOX, and also by curbing LPS-triggered reactive oxygen species generation (32). While 11(R)-HETE can be formed by both COX isoforms $(7,33)$, it was induced by LPS in plasma, unlike 12-HHT and 12(S)-HETE, and suppressed by COX-2i in vitro, all of which is consistent with it being derived predominantly from COX-2. The failure of celecoxib to reduce 11(R)-HETE ex vivo may reflect the variance between individuals relative to the signal, even when adjusted for their own controls. Overall, the suppressive effects of the COX-2i on 15- and 11-HETEs in human whole blood are marks of distinction from the lipidomic effects of mPGES inhibition.

Expression of the 5-LOX protein coincides with FLAP, $\mathrm{LTA}_{4} \mathrm{H}$, and $\mathrm{LTC}_{4} \mathrm{~S}$ and is restricted to cells of myeloid origin, including neutrophils, eosinophils, monocytes/macrophages, and mast cells (19). In whole blood, neutrophils are the primary source of LTs and 5-HETE; peripheral blood mononuclear cells and, to a lesser degree, eosinophils may also contribute (34). Zymosan is a naturally occurring proinflammatory ligand that elevates intracellular calcium and thus triggers LT synthesis in cells possessing the 5-LOX 
enzyme (35). Although LPS does not directly activate 5-LOX synthesis, LPS can prime neutrophils for increased production of $\mathrm{LTB}_{4}$ in response to subsequent stimulation with zymosan (36). Thus, it is possible that costimulation of whole blood with LPS and zymosan in our study potentiated the capacity of neutrophils to produce LTs. Platelets and red blood cells do not possess 5-LOX or FLAP, but they contain secondary enzymes, such as $\mathrm{LTA}_{4} \mathrm{H}$ or $\mathrm{LTC}_{4} \mathrm{~S}$, and may contribute to LT production through transcellular biosynthesis (18). Despite the capacity-related feature of this assay, with provocation by inflammatory stimuli, only modest amounts of $\mathrm{LXA}_{4}$ and RvE1 were evident, while most specialized proresolving mediators were not detected. We and others have also largely failed to detect endogenous production of these mediators in plasma or urine $(37,38)$ and failed to relate alterations in specialized proresolving mediator formation in vivo in response to the evocation of inflammation with LPS or its resolution or the dose-dependent provision of their theoretical substrate (38).

Expression of data as a percentage of a subject's own stimulus control permits assessment of drug effect on the plasma lipidome in small sample sizes, despite variability in absolute values between donors. Standardization of data for individual responses lets us capture evidence consistent with substrate rediversion during screening of drugs targeting mPGES-1 or LTA 4 . Substrate shunting or rediversion may occur when inhibition of one terminal synthase makes more of the common substrate $\mathrm{PGH}_{2}$ available for other PG synthases (Figure 1). Targeting of a terminal PG synthase can also alter AA substrate availability to other pathways. Thus, we observed that blockade of mPGES-1 inhibited PGE ${ }_{2}$ production but elevated products of PGFS, TxS, and, potentially, 15-LOX. Similarly, LTA 4 substrate may be shunted between $\mathrm{LTA}_{4} \mathrm{H}$ and $\mathrm{LTC}_{4} \mathrm{~S}$ in the 5-LOX pathway (Figure 1). Here, inhibition of $\mathrm{LTA}_{4} \mathrm{H}$ resulted in reduced levels of $\mathrm{LTB}_{4}$ but augmentation of $\mathrm{LTC}_{4} \mathrm{~S}$ product $\mathrm{LTE}_{4}$.

Considering the involvement of both COX and LOX pathways in common inflammatory diseases, such as atherosclerosis $(13,14)$, abdominal aortic aneurysm (39-41), asthma $(42,43)$, arthritis, and inflammatory pain $(13,44,45)$, dual inhibitors of both pathways are of interest (46). Concurrent blockade of mPGES-1 with inhibition of various components of the 5-LOX pathway revealed both predicted and unexpected effects on the human plasma lipidome. Coincubations of an mPGES-1i with inhibitors of FLAP or $\mathrm{LTA}_{4} \mathrm{H}$ led to initial shunting of the substrate toward 12-LOX and possibly 15-LOX, with a subsequent rediversion toward the COX pathway (Figure 4). In the combination with the FLAPi, sustained inhibition of 5-HETE, LTs, and $\mathrm{PGE}_{2}$ coincided with a concurrent increase in $\mathrm{PGF}_{2 \alpha}$ and $\mathrm{TxB}_{2}$, characteristic of the mPGES-1i. Thus, each drug retained features of their distinct mechanism of action when combined in whole blood. Surprisingly, when the mPGES-1i was combined with the 5-LOXi, which would be expected to recapitulate the effect of FLAP inhibition, there was early suppression of both PGs and 5-LOX products, with later reductions in total 12-HETE and 15-HETE but no evidence of substrate rediversion. Thus, combination of the mPGES-1i with compounds that have apparently similar mechanisms of action, FLAP and 5-LOX inhibitors, has distinct effects on the plasma lipidome.

In summary, using a small-scale in vitro whole-blood assay and broad-spectrum lipidomics analysis, we can study drug effects on the COX and LOX pathways in plasma samples of limited quantity. The method complements in vivo analysis of eicosanoid metabolites and MS-based analysis of target modification, such as acetylation of COX-1 by aspirin (47). It can be applied to in vitro screening of drug combinations and can be readily extended for the quantitation of other eicosanoids, as the system is applicable to lipid metabolites with diverse hydrophobicity.

\section{Methods}

Reagents. Deuterated analogs of $\mathrm{PGE}_{2}, \mathrm{PGD}_{2}, \mathrm{PGF}_{2 \alpha}, 6$-keto-PGF $\left(\right.$ the hydrolysis product of $\mathrm{PGI}_{2}$ ), $\mathrm{TxB}_{2}$ (the hydrolysis product of TxA ${ }_{2}$ ), LTE LTB $_{4}, 5$-HETE, 12-HETE, 15-HETE, 20-HETE, AA, EPA, DHA, 7-hydroxy-5,11-diketotetranorprostane-1,16-dioic acid (PGE-M), 11,15-dioxo-9 $\alpha$ hydroxy-, 2,3,4,5-tetra-

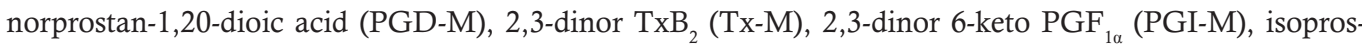
tane 8,12-iso-iPF ${ }_{2 \alpha}$-VI, and creatinine and authentic standards for 11(S)-HETE, 11(R)-HETE, ( \pm )12-HETE, and $( \pm)$ 15-HETE were purchased from Cayman Chemical $\left(\left[\mathrm{d}_{4}\right]-\mathrm{PGE}_{2},\left[\mathrm{~d}_{4}\right]-\mathrm{PGF}_{2 \alpha},\left[\mathrm{d}_{4}\right]-\mathrm{TxB}_{2},\left[\mathrm{~d}_{4}\right]-\mathrm{LTB}_{4}\right.$, $\left[\mathrm{d}_{5}\right]$-LTE $4,\left[\mathrm{~d}_{8}\right]-5$-HETE, $\left[\mathrm{d}_{8}\right]$-12-HETE, $\left[\mathrm{d}_{8}\right]$-15-HETE, $\left[\mathrm{d}_{8}\right]$-AA, $\left[\mathrm{d}_{5}\right]$-EPA, [ $\left.\mathrm{d}_{5}\right]$-DHA, [d 6 -tetranor-PGEM, $\left[\mathrm{d}_{6}\right]$-tetranor-PGDM, $\left[\mathrm{d}_{3}\right]$-2,3-dinor-6-keto-PGF ${ }_{1 \alpha}, \quad\left[\mathrm{d}_{4}\right]$-11-dehydro-TxB $, \quad\left[\mathrm{d}_{11}\right]-8,12-$-iso-iPF $2 \alpha-\mathrm{VI}, \quad$ and $\left[\mathrm{d}_{3}\right]$-creatinine). Stock solutions were prepared in acetonitrile and stored in glass vials at $-80^{\circ} \mathrm{C}$. We utilized purified deionized water (Milli-Q water purification system, EMD Millipore) in the preparation of all aqueous solutions and mobile phases. UPLC-grade acetonitrile and ethyl acetate were purchased from 
J. T. Baker. Reagent-grade acetic acid, formic acid, isopropanol, and ethanol were purchased from Fisher Scientific. Methoxyamine hydrochloride (MO HCL), diisopropylethylamine (DIPEA), 2,3,4,5,6-pentafluorobenzyl bromide (PFB-Br), and charcoal stripped FBS were purchased from Sigma-Aldrich. Gases were supplied by BOC Gases. Bacterial LPS derived from E. coli 0111:B4 (L2630) and Zymosan A derived from Saccharomyces cerevisiae (Z4250) were purchased from Sigma-Aldrich. DPBS was obtained from Life Technologies; DMSO came from Sigma-Aldrich. Heparin sodium (1,000 U/ml) was purchased from Patterson Veterinary. Drugs and compounds for the in vitro assay (COX-2i celecoxib, ref. 48; COX-1/-2i diclofenac, ref. 49; COX-1/-2i aspirin, ref. 49; COX-2i GW406381, ref. 50; mPGES-1i MF-63, ref. 51; PGE 2 receptor EP4 antagonist CJ-042794, ref. 52; 5-LOXi ABT-761, ref. 53 (atreleuton); FLAPi MK-0591, ref. 54; LTA $_{4}$ Hi SC-57461A, ref. 55; BLT1-selective LTB $_{4}$ receptor antagonist LY293111, ref. 56 (etalocib); and BLT2-selective $\mathrm{LTB}_{4}$ receptor antagonist LY255283, refs. 57, 58) were provided by Eli Lilly \& Co through the courtesy of M.J. Fisher (Eli Lilly \& Co, Indianapolis, Indiana). Celecoxib and placebo pills for the ex vivo study were provided by the Investigational Drug Service of the University of Pennsylvania.

Clinical studies. Two clinical studies were performed (Clinical Trials.gov NCT02095288 and NCT02413203). All volunteers (age 18-50 years old) were apparently healthy on physical examination, were nonsmokers and nonpregnant, and were required to abstain from all medications, including NSAIDs, for at least 2 weeks before enrollment. Volunteers with a history of coagulation or bleeding disorders, drug allergies, or gastrointestinal diseases were excluded from participation in the studies.

For the in vitro whole blood assay, 19 fasted volunteers ( 7 male and 12 female) provided peripheral venous blood samples between 0900 and 1000 hours on the day of the visit. For the validation of the in vitro assay in an ex vivo setting, a randomized, placebo-controlled, double-blind study was performed. Twenty volunteers (13 male and 7 female) were randomly assigned by the Investigational Drug Service to receive celecoxib $(n=10)$ or placebo $(n=10)$. Each subject received a single, 200-mg oral dose of celecoxib or placebo. The drugs were administered to the fasted volunteers between 0900 and 0930 hours on the study morning. Blood and urine collections were performed before (pre-dose) and 3 hours after (post-dose) the drug administration, which corresponds to $\mathrm{T}_{\max }$ for celecoxib (the time at which celecoxib plasma level is maximum). Volunteers remained at the Clinical and Translational Research Center for the duration of the study. Human whole-blood assay was performed on pre- and post-dose blood samples. Systemic effects of the drug treatment on eicosanoids were assessed by analysis of urinary metabolites.

Human whole-blood assay. Human whole blood was collected by venipuncture. For single-compound screening, all the drugs, except aspirin, were screened at $\mathrm{IC}_{50}$ s reported previously (59) (Supplemental Table 1) and at an absolute concentration of $10 \mu \mathrm{M}$. Aspirin was screened at $15 \mu \mathrm{M}$, corresponding to the peak systemic plasma levels after ingestion of a low dose (59), and at $1.5 \mathrm{mM}$, a rather high concentration, at which both COX isoforms would be expected to be inhibited by the drug. For screening of the COX pathway inhibitors, $500 \mu 1$ of whole blood containing $10 \mathrm{IU}$ sodium heparin was incubated with $2 \mu 1$ of a test compound or vehicle (DMSO) for 30 minutes at $37^{\circ} \mathrm{C}$ in a 96-deep-well, sterile, polypropylene plate (Thermo Scientific, catalog 260251). Plates were covered with MicroClime-Environmental lids (Labcyte, catalog LLS-0310) to minimize edge effects. Then, $10 \mu \mathrm{LPS}$ in PBS (100 $\mu \mathrm{g} / \mathrm{ml}$ final concentration) were added to the blood and incubated for additional 24 hours. After the incubation, blood was spun down at 3,000 $g$ for 10 minutes at $4^{\circ} \mathrm{C}$ and plasma was isolated for LC-MS analysis. For screening of the LOX pathway inhibitors, $500 \mu 1$ heparinized blood was incubated with $10 \mu 1$ zymosan in PBS $(125 \mu \mathrm{g} / \mathrm{ml}$ final concentration) for 4 hours in the presence of $2 \mu \mathrm{DMSO}$ or a test compound, which was added 30 minutes before the addition of zymosan. Blood samples, spun down in polypropylene tubes (Sarstedt, $5 \mathrm{ml}, 75 \times 12 \mathrm{~mm}$, Fisher Scientific) immediately after the blood draw, served as untreated controls. For drug combinations, a compound-targeting COX pathway was coincubated with a compound targeting the 5-LOX pathway. Thus, COX-2i celecoxib, mPGES-1i MF-63, and EP4 antagonist CJ-042794 were coincubated, each with the 5-LOXi ABT-761, the FLAPi MK-0591, or the LTA Hi SC-57461A. To ensure a concurrent, maximum activation of the COX and 5-LOX pathways, optimal stimulating conditions for each of these pathways alone were combined in drug combination screening experiments. Thus, whole blood containing two types of inhibitors was simulated with both LPS and zymosan at 4 and 24 hours. Each test compound was delivered in $1 \mu \mathrm{DMSO}$ to the well bottom, avoiding mixing of the drugs, to the final concentration of $10 \mu \mathrm{M}$ for each compound. Then, $500 \mu 1$ of heparinized blood was added to the well and incubated for 30 minutes at $37^{\circ} \mathrm{C}$. After that, $5 \mu \mathrm{LPS}$ and $5 \mu \mathrm{zymosan}$ were added to the final concentrations of $100 \mu \mathrm{g} / \mathrm{ml}$ and $125 \mu \mathrm{g} / \mathrm{ml}$, respectively, and incubated for 4 and 24 hours. Drug combinations stimulated with either LPS 
or zymosan alone served as internal controls for stimulating conditions. For the in vitro assay, data were normalized to plasma volume and expressed as a percentage of patient's stimulus + DMSO control using the formula: percentage of stimulus control $=\left(C_{\text {sample }} / C_{\text {stimulus }}\right) \times 100 \%$, where $C$ represents eicosanoid concentration in $\mathrm{ng} / \mathrm{ml}$. For the ex vivo assay, data were normalized to plasma volume and expressed as a percentage of patient's pre-dose control using the formula: percentage of pre-dose control $=\left(C_{\text {post-dose }} / C_{\text {pre-dose }}\right)$ $\times 100 \%$, where $C$ represents eicosanoid concentration in $\mathrm{ng} / \mathrm{ml}$.

Analytical method development for broad-spectrum eicosanoid quantification in human plasma. A UPLC-MS/ MS assay was developed to measure a wide range of COX and LOX products in plasma in a single run. In addition to $\mathrm{PGE}_{2}$, inhibition of which contributes substantially to the therapeutic effects of NSAIDs (13), we were interested in other COX products as possible products of substrate rediversion after mPGES-1 inhibition in whole blood. Thus, we focused on $\mathrm{PGD}_{2}, \mathrm{PGF}_{2 \alpha}, 6-\mathrm{keto}-\mathrm{PGF}_{1 \alpha}, \mathrm{TxB}_{2}$, and 12-HHT. Considering that substrate shifts could occur beyond the COX pathway, for instance, toward LOX or cytochrome P450 (CYP) cascades, we measured 5-HETE and $\mathrm{LTB}_{4}$ and $\mathrm{LTE}_{4}$ as indices of 5-LOX activation as well as 12-HETE and 15-HETE, products of 12- and 15-LOX, respectively, and 20-HETE, an AA-derived metabolite made by CYP enzymes. We also measured resolvins (RvD1, RvD2, RvE1), lipoxin $\mathrm{A}_{4}$, maresin 1, and protectin. We measured AA levels to assess how different stimulating conditions and inhibition with drugs affect the substrate in the whole-blood matrix. Finally, we were interested to determine whether activation of blood cells affected plasma levels of EPA and DHA. Thus, our initial mix of standards contained 17 compounds to monitor 21 analytes. Extensive kinetics (0, 0.5, 1, 2, 4, 8, 24, 32, and 48 hours) and stimulus dose-response experiments were done to establish optimal conditions for activation of COX and LOX pathways in human whole blood. For COX activation, we selected bacterial endotoxin LPS, a known trigger of PG production in whole blood $(60,61)$. For LOX activation, we selected a yeast-derived TLR2 agonist, zymosan. Once optimal conditions for COX and LOX pathways were determined, the spike was reduced to 9 standards.

UPLC-MS/MS analysis of plasma samples. The analysis was performed on a Waters ACQUITY UPLC system in-line with a Waters Xevo TQ-S Triple Quadrupole Mass Spectrometer.

Plasma samples $(200 \mu \mathrm{l})$ were spiked with stable isotope-labeled internal standards $\left(\left[\mathrm{d}_{4}\right]-\mathrm{PGE}_{2}[5\right.$ $\mathrm{ng}] ;\left[\mathrm{d}_{4}\right]-\mathrm{PGF}_{2 \alpha}[2.5 \mathrm{ng}] ;\left[\mathrm{d}_{4}\right]-\mathrm{TxB}_{2}[10 \mathrm{ng}] ;\left[\mathrm{d}_{4}\right]-\mathrm{LTB}_{4}[1 \mathrm{ng}] ;\left[\mathrm{d}_{5}\right]-\mathrm{LTE}_{4}[2.5 \mathrm{ng}] ;\left[\mathrm{d}_{8}\right]-5-\mathrm{HETE}[2.5 \mathrm{ng}] ;$

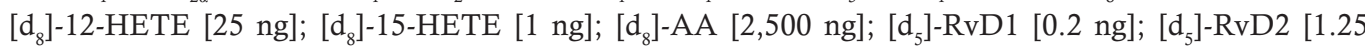
$\left.\mathrm{ng}] ;\left[\mathrm{d}_{5}\right]-\mathrm{LXA}_{4}[1 \mathrm{ng}]\right)$ in $900 \mu \mathrm{l}$ of acetonitrile. Then, they were acidified by addition of formic acid to a final concentration of $1 \%$. The samples were vortexed, incubated at room temperature for 15 minutes, vortexed again, and sonicated for 1 minute. After that, they were spun down, and the supernatant was transferred to Phree cartridges for phospholipid and protein removal (Phenomenex, 8B-S133-TAK). The samples were eluted with a slight vacuum $(<20 \mathrm{kPa})$. They were then dried under a gentle stream of nitrogen at the ambient temperature and reconstituted with $25 \mu 1$ of methanol.

Before injection, $25 \mu 1$ of water was added to each sample and an aliquot of $20 \mu 1$ was injected into a C18 UPLC column (Waters ACQUITY UPLC BEH $2.1 \times 150 \mathrm{~mm} \times 1.7 \mu \mathrm{m}$ ) and eluted at a flow rate of $350 \mu 1 / \mathrm{min}$, with a linear gradient from $20 \%$ solvent B to $90 \%$ in 20 minutes. Mobile-phase and MS parameters were optimized to obtain maximum sensitivity for respective product ions. Mobile phase $\mathrm{A}$ consisted of water/mobile phase B, 95:5 (v/v), with 0.5\% formic acid; mobile phase B consisted of acetonitrile/methanol, $95: 5$ (v/v), with $0.5 \%$ formic acid. The UPLC system was directly interfaced with the negative-mode electrospray ionization (ESI) source of the mass spectrometer using multiple reaction monitoring (MRM) of all transitions (Supplemental Table 2). A single run took 26 minutes. Quantitation was done by peak area ratio, and results were normalized to plasma volume.

$L C-M S / M S$ analysis of urine samples. Systemic urinary production of $\mathrm{PGE}_{2}, \mathrm{PGD}_{2}, \mathrm{TxA}_{2}$, and $\mathrm{PGI}_{2}$ was determined by quantification of their major urinary metabolites (PGE-M, PGD-M, Tx-M, PGI-M), and isoprostane $8,12-i s o-\mathrm{PF}_{2 \alpha}$-VI was measured as an index of lipid peroxidation. Stable isotope-labeled

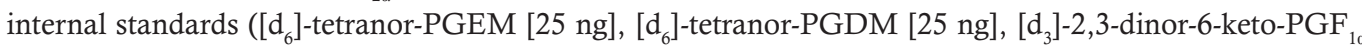
[5 ng], [ $\left.\mathrm{d}_{4}\right]$-11-dehydro- $\mathrm{TxB}_{2}[5 \mathrm{ng}],\left[\mathrm{d}_{11}\right]-8,12-$-iso-iPF $\left.{ }_{2 \alpha}-\mathrm{VI}[5 \mathrm{ng}]\right)$ were added to $1 \mathrm{ml}$ of human urine in 50 $\mu 1$ of acetonitrile and allowed to equilibrate for 15 minutes. $500 \mu \mathrm{l}$ of $\mathrm{MO} \mathrm{HCl}$ was added and allowed to equilibrate at $\mathrm{pH} \approx 3$ for 30 minutes. The samples were purified by solid-phase extraction using Strata-X 33 $\mu \mathrm{m}$ polymeric reversed phase, $30 \mathrm{mg} / \mathrm{ml}$ cartridges (Phenomenex, 8B-S100-TAK). The SPE cartridge was conditioned with $1 \mathrm{ml}$ of acetonitrile and equilibrated with $0.25 \mathrm{ml}$ of water. The sample was applied to the cartridge, which was then washed with $1 \mathrm{ml}$ of water and dried with a vacuum for 15 minutes. The analyte 
and internal standards were eluted from the cartridge using $1 \mathrm{ml}$ of $5 \%$ acetonitrile in ethyl acetate. The eluate was collected and dried under a gentle stream of nitrogen. The resulting residue was then reconstituted in $180 \mu 1$ of $5 \%$ acetonitrile in water, and $75 \mu 1$ was transferred into an autosampler vial and injected into the HPLC-MS/MS system. A Shimadzu Prominence UPLC system was used for chromatography. The UPLC column was $2.1 \times 150 \mathrm{~mm}$, with $1.7-\mu \mathrm{m}$ particles (Waters ACQUITY UPLC CSH C18). Mobile phase A consisted water/mobile phase B, 95:5 (v/v), containing 1\% acetic acid adjusted to $\mathrm{pH} 5.7$ with ammonium hydroxide. Mobile phase B consisted of acetonitrile/methanol, 95:5 (v/v). The flow rate was $350 \mu 1 / \mathrm{min}$. Separations were carried out with various linear solvent gradients. The Thermo Finnigan TSQ Quantum Ultra tandem instrument (Thermo Fisher Scientific) equipped with a triple quadrupole analyzer was operated in negative-mode ESI, and the analyzer was set in the MRM mode for the analysis of the urinary metabolites. To adjust for urine production, data were normalized to creatinine levels. Here, $20 \mu 1$ of urine was added to $1 \mathrm{ml}$ of $\left[\mathrm{d}_{3}\right]$-creatinine in $\mathrm{AcN}$. Next, $40 \mu \mathrm{l}$ of spiked urine was further diluted with $360 \mu \mathrm{l}$ of $\mathrm{AcN}$, and $20 \mu \mathrm{l}$ of the sample was injected into a HPLC column (Waters XBridge BEH HILIC $2.1 \times 50 \mathrm{~mm} \times 2.5 \mu \mathrm{m}$ ). Samples were eluted at a flow rate of $350 \mu 1 / \mathrm{min}$ with an isocratic $12 \%$ solvent $\mathrm{B}$ in 1.5 minutes. Mobile phase A was $100 \%$ AcN; mobile phase B consisted of $5 \mathrm{mM}$ ammonium formate ( $\mathrm{pH}$ =4). The HPLC system (Thermo Scientific Accela Pump) was directly interfaced with the positive-mode ESI source of the mass spectrometer (Thermo Electron Corporation TSQ Quantum U1tra) using MRM of endogenous creatinine $(\mathrm{m} / \mathrm{z} 114.0>86.0 \mathrm{amu}, 15.0 \mathrm{eV})$ and $\left[\mathrm{d}_{3}\right]$-creatinine $(\mathrm{m} / \mathrm{z} 117.0>89.0 \mathrm{amu}, 15.0$ $\mathrm{eV})$. Urine data are reported as a percentage of the volunteer's own pre-dose control using the formula: percentage of pre-dose control $=\left(C_{\text {post-dose }} / C_{\text {pre-dose }}\right) \times 100 \%$, where $C$ represents metabolite concentration in $\mathrm{ng} / \mathrm{mg}$ creatinine.

Chiral UPLC-electron capture atmospheric pressure chemical ionization/high-resolution MS analysis. Chiral products of 8-HETE, 11-HETE, 12-HETE, and 15-HETE were quantified by a targeted chiral lipidomics approach using UPLC-electron capture atmospheric pressure chemical ionization/high-resolution MS (ECAPCI/HRMS) methodology based on a technique reported previously with a Thermo Scientific Q-Exactive HF high-resolution mass spectrometer replacing the triple quadrupole instrument (62). The pentafluorobenzyl (PFB) derivatives of 8 lipids and a heavy isotope analog internal standard of 15(S)-HETE were prepared by dissolving the residues from extracted plasma in $100 \mu 1$ diisopropylethylamine in acetonitrile $(1: 19, \mathrm{v} / \mathrm{v})$ followed by $50 \mu \mathrm{PFB}-\mathrm{Br}$ in acetonitrile $(1: 9, \mathrm{v} / \mathrm{v})$. The solution was incubated at $60^{\circ} \mathrm{C}$ for 30 minutes, allowed to cool, evaporated to dryness under a nitrogen stream at room temperature, and redissolved in $100 \mu \mathrm{l}$ hexane/ethanol (97:3, v/v) for chiral UPLC-HRMS analysis. A 5- $\mu$ l aliquot was injected for each sample. Normal-phase chiral chromatography was performed using an UltiMate 3000 binary UPLC equipped with a refrigerated autosampler $\left(6^{\circ} \mathrm{C}\right)$ and a column heater $\left(35^{\circ} \mathrm{C}\right)$. Gradient elution was performed in the linear mode. A Chiralpak AD-H column $(250 \times 4.6 \mathrm{~mm}$ i.d., $5 \mu \mathrm{m}$; Chiral Technologies $)$ was employed with a flow rate of $1 \mathrm{ml} / \mathrm{min}$. Solvent A was hexane and solvent B was 2-propanol $/ \mathrm{methanol}$ $(5: 5, \mathrm{v} / \mathrm{v})$. The linear gradient was as follows: $2 \% \mathrm{~B}$ at 0 minutes, $2 \% \mathrm{~B}$ at 3 minutes, $8 \% \mathrm{~B}$ at 11 minutes, $8 \%$ $\mathrm{B}$ at 13 minutes, $95 \% \mathrm{~B}$ at 17 minutes, $2 \% \mathrm{~B}$ at 18 minutes, and $2 \% \mathrm{~B}$ at 22 minutes. MS was conducted on a Thermo Scientific Q-Exactive HF Hybrid Quadrupole-Orbitrap mass spectrometer. The column effluent was diverted to waste 3 minutes before and 14 minutes after the run. The mass spectrometer was equipped with an APCI source in the negative electron-capturing ion mode (62). The operating conditions were as follows: vaporizer temperature, $450^{\circ} \mathrm{C}$; heated capillary temperature, $320^{\circ} \mathrm{C}$; corona discharge needle, set at $30 \mu \mathrm{A}$. The sheath gas (nitrogen) and auxiliary gas (nitrogen) pressures were 40 psi and 10 arbitrary units, respectively. The S-lens was set at 60 . The Q-Exactive-HF instrument was alternating between a full scan $(100-600 \mathrm{~m} / z)$ at a resolution of 30,000 and parallel reaction monitoring (PRM) at 60,000 resolution with a precursor isolation window of $2 \mathrm{~m} / z$ and normalized collision energy of 20\%. Molecular (M-PFB) precursor ions for 15-HETE and [ $\left.\mathrm{d}_{8}\right]-15-(S)$-HETE were 319.2323 and 327.2781, respectively. The most intense product ions for 15-HETE and [ $\left.\mathrm{d}_{8}\right]$-15-(S)-HETE were 216.1372 and 226.1815, accordingly. The PRM transitions for all chiral products are summarized in Supplemental Table 2. Quantification was based on the product ion with $m / z \pm 3$ ppm of theoretical. Data analysis was performed using Xcalibur software, version 2.0 SR2 (Thermo Scientific), from raw mass spectral data. Calibration standard samples were prepared with charcoal-stripped FBS. Calibration samples were spiked with authentic standards of 11(S)-HETE, 11(R)-HETE, $( \pm) 12$-HETE, and $( \pm) 15$-HETE in the amounts of $0,0.1,0.25,0.5,1,2.5,5 \mathrm{ng}$, and $1 \mathrm{ng}$ of the internal standard $\left[\mathrm{d}_{8}\right]-15-(S)$-HETE. Oxidized lipids were extracted, purified, derivatized, and analyzed as described above for the analytical samples. Calibration curves were plotted using a linear regression of 
peak area ration of analytes against internal standard (Supplemental Figure 12). Typical regression lines for 11( $R$ )-HETE, 11(S)-HETE, 12( $R$ )-HETE, 12(S)-HETE, 15( $R$ )-HETE, and 15( $S)$-HETE were $y=4.60723 x$ $+1.46655\left(r^{2}=0.9959\right), y=4.44665 x+1.38262\left(r^{2}=0.9914\right), y=1.09631 x+0.748153\left(r^{2}=0.9959\right), y=$ $1.06212 x+1.09477\left(r^{2}=0.9850\right), y=0.338872 x+0.189865\left(r^{2}=0.9955\right)$, and $y=0.752994 x+0.393947$ $\left(r^{2}=0.9986\right)$, respectively. Concentrations of the chiral products were calculated by interpolation from the calculated regression lines.

Statistics. Statistical analyses were performed using GraphPad Prism software version 5.0 for Mac OS $\mathrm{X}$. The statistical significance of the differences between various treatments was determined by 1 -sample, 2-tailed $t$ tests for the in vitro assays and by unpaired, 2-tailed $t$ tests for the ex vivo assay. $P<0.05$ was considered significant. Plots for plasma and urine data from the ex vivo study were built using GraphPad Prism software. The investigators were blinded to sample allocation during the ex vivo study and outcome assessment. Visualization of broad-spectrum lipidomic changes in in vitro stimulated and drug-treated whole blood was generated using the open-source Circos software package (63).

Study approval. All clinical studies were conducted according to Declaration of Helsinki principles. The study protocols were approved by the Institutional Review Board of the University of Pennsylvania and by the Advisory Council of the Clinical and Translational Research Center of the University of Pennsylvania. All study volunteers provided written informed consent prior to inclusion in the study.

\section{Author contributions}

LLM, TG, ER, and GAF designed the study and experiments. LLM, ER, and GAF analyzed data. LLM performed the experiments and coordinated the clinical studies. JAL developed the LC-MS method and analyzed MS data for the in vitro study. XL analyzed MS data for drug combinations experiments and the ex vivo study. CM and IAB developed and performed chiral UPLC-ECAPCI/HRMS analyses. GG created Circos plots. LLM and GAF wrote the manuscript.

\section{Acknowledgments}

We thank M.J. Fisher, A. Harvey, S. Chandrasekhar, and A.M. Warshawsky (Eli Lilly \& Co, Indianapolis, Indiana, USA) for valuable discussion and providing test compounds. We thank Helen Zou and Wenxuan Li for technical support. This study was supported by a grant from Eli Lilly \& Co, The Personalized NSAID Therapeutics Consortium (PENTACON: HL117798), grants from the NIH (T32 H107971 to LLM and UL1TR000003), and the National Center for Research Resources and the National Center for Advancing Translational Sciences. The content is solely the responsibility of the authors and does not necessarily represent the official views of the NIH. GAF is the McNeil Professor in Translational Medicine and Therapeutics.

Address correspondence to: Garret A. FitzGerald, Institute for Translational Medicine and Therapeutics, Smilow Center for Translational Research, Room 10-122, 3400 Civic Center Boulevard, Building 421, Philadelphia, Pennsylvania 19104-5158, USA. Phone: 215.898.1185; E-mail: garret@upenn.edu.

\footnotetext{
1. Lerner MR, et al. Tools for investigating functional interactions between lipid-derived autacoids and their receptors. Am $J$ Ther 1996;3(4):280-286.

2. Fitzgerald DJ, Fitzgerald GA. Historical lessons in translational medicine: cyclooxygenase inhibition and P2Y12 antagonism. Circ Res. 2013;112(1):174-194.

3. Grosser T, Yu Y, Fitzgerald GA. Emotion recollected in tranquility: lessons learned from the COX-2 saga. Annu Rev Med. 2010;61:17-33.

4. Cheng Y, Wang M, Yu Y, Lawson J, Funk CD, Fitzgerald GA. Cyclooxygenases, microsomal prostaglandin E synthase-1, and cardiovascular function. J Clin Invest. 2006;116(5):1391-1399.

5. Catella F, FitzGerald GA. Paired analysis of urinary thromboxane B2 metabolites in humans. Thromb Res. 1987;47(6):647-656.

6. Alessandrini P, Avogaro P, Bittolo Bon G, Patrignani P, Patrono C. Physiologic variables affecting thromboxane B2 production in human whole blood. Thromb Res. 1985;37(1):1-8.

7. Lee SH, Williams MV, Dubois RN, Blair IA. Cyclooxygenase-2-mediated DNA damage. J Biol Chem. 2005;280(31):28337-28346.

8. Hecker M, Ullrich V, Fischer C, Meese CO. Identification of novel arachidonic acid metabolites formed by prostaglandin H synthase. Eur J Biochem. 1987;169(1):113-123.

9. Porter NA, Caldwell SE, Mills KA. Mechanisms of free radical oxidation of unsaturated lipids. Lipids. 1995;30(4):277-290.

10. Haeggström JZ, Funk CD. Lipoxygenase and leukotriene pathways: biochemistry, biology, and roles in disease. Chem Rev. 2011;111(10):5866-5898.

11. Zarini S, Gijón MA, Ransome AE, Murphy RC, Sala A. Transcellular biosynthesis of cysteinyl leukotrienes in vivo during
} 
mouse peritoneal inflammation. Proc Natl Acad Sci U S A. 2009;106(20):8296-8301.

12. Fabre JE, et al. Transcellular biosynthesis contributes to the production of leukotrienes during inflammatory responses in vivo. J Clin Invest. 2002;109(10):1373-1380.

13. Ricciotti E, FitzGerald GA. Prostaglandins and inflammation. Arterioscler Thromb Vasc Biol. 2011;31(5):986-1000.

14. Zhao L, Funk CD. Lipoxygenase pathways in atherogenesis. Trends Cardiovasc Med. 2004;14(5):191-195.

15. Martel-Pelletier J, Lajeunesse D, Reboul P, Pelletier JP. Therapeutic role of dual inhibitors of 5-LOX and COX, selective and non-selective non-steroidal anti-inflammatory drugs. Ann Rheum Dis. 2003;62(6):501-509.

16. Fries S, et al. Marked interindividual variability in the response to selective inhibitors of cyclooxygenase-2. Gastroenterology. 2006;130(1):55-64.

17. McAdam BF, Catella-Lawson F, Mardini IA, Kapoor S, Lawson JA, FitzGerald GA. Systemic biosynthesis of prostacyclin by cyclooxygenase (COX)-2: the human pharmacology of a selective inhibitor of COX-2. Proc Natl Acad Sci U S A. 1999;96(1):272-277.

18. Capra V, Rovati GE, Mangano P, Buccellati C, Murphy RC, Sala A. Transcellular biosynthesis of eicosanoid lipid mediators. Biochim Biophys Acta. 2015;1851(4):377-382.

19. Rådmark O, Werz O, Steinhilber D, Samuelsson B. 5-Lipoxygenase, a key enzyme for leukotriene biosynthesis in health and disease. Biochim Biophys Acta. 2015;1851(4):331-339.

20. Nichols FC, Schenkein HA, Rutherford RB. Prostaglandin E2, prostaglandin E1 and thromboxane B2 release from human monocytes treated with C3b or bacterial lipopolysaccharide. Biochim Biophys Acta. 1987;927(2):149-157.

21. Fu JY, Masferrer JL, Seibert K, Raz A, Needleman P. The induction and suppression of prostaglandin H2 synthase (cyclooxygenase) in human monocytes. J Biol Chem. 1990;265(28):16737-16740.

22. Zheng H, Crowley JJ, Chan JC, Raffin TA. Attenuation of LPS-induced neutrophil thromboxane b2 release and chemiluminescence. J Cell Physiol. 1991;146(2):264-269.

23. Niiro H, et al. Regulation by interleukin-10 and interleukin-4 of cyclooxygenase-2 expression in human neutrophils. Blood. 1997;89(5):1621-1628.

24. Fasano MB, Wells JD, McCall CE. Human neutrophils express the prostaglandin G/H synthase 2 gene when stimulated with bacterial lipopolysaccharide. Clin Immunol Immunopathol. 1998;87(3):304-308.

25. Pouliot M, et al. Expression and activity of prostaglandin endoperoxide synthase-2 in agonist-activated human neutrophils. FASEB J. 1998;12(12):1109-1123.

26. Rousson D, Lagarde M, Touboul P, Dechavanne M. Acute myocardial infarction: measurement of arachidonate end-products in whole blood as an index of platelet cyclo-oxygenase activity in vivo. Thromb Res. 1987;48(1):63-71.

27. Okuno T, Iizuka Y, Okazaki H, Yokomizo T, Taguchi R, Shimizu T. 12(S)-Hydroxyheptadeca-5Z, 8E, 10E-trienoic acid is a natural ligand for leukotriene B4 receptor 2. J Exp Med. 2008;205(4):759-766.

28. Brash AR, Murray JJ, Oates JA. The 5-lipoxygenase and 15-lipoxygenase of neutrophils and eosinophils. Prog Clin Biol Res. 1985;199:143-152.

29. Nassar GM, Morrow JD, Roberts LJ, Lakkis FG, Badr KF. Induction of 15-lipoxygenase by interleukin-13 in human blood monocytes. J Biol Chem. 1994;269(44):27631-27634.

30. Turk J, Maas RL, Brash AR, Roberts LJ, Oates JA. Arachidonic acid 15-lipoxygenase products from human eosinophils. J Biol Chem. 1982;257(12):7068-7076.

31. Levy BD, Clish CB, Schmidt B, Gronert K, Serhan CN. Lipid mediator class switching during acute inflammation: signals in resolution. Nat Immunol. 2001;2(7):612-619.

32. Nakamura Y, et al. Arachidonic acid cascade inhibitors modulate phorbol ester-induced oxidative stress in female ICR mouse skin: differential roles of 5-lipoxygenase and cyclooxygenase-2 in leukocyte infiltration and activation. Free Radic Biol Med. 2003;35(9):997-1007.

33. Jian W, Lee SH, Williams MV, Blair IA. 5-Lipoxygenase-mediated endogenous DNA damage. J Biol Chem. 2009;284(25):1679916807.

34. Surette ME, Palmantier R, Gosselin J, Borgeat P. Lipopolysaccharides prime whole human blood and isolated neutrophils for the increased synthesis of 5-lipoxygenase products by enhancing arachidonic acid availability: involvement of the CD14 antigen. J Exp Med. 1993;178(4):1347-1355.

35. Werz O. 5-lipoxygenase: cellular biology and molecular pharmacology. Curr Drug Targets Inflamm Allergy. 2002;1(1):23-44.

36. Doerfler ME, Danner RL, Shelhamer JH, Parrillo JE. Bacterial lipopolysaccharides prime human neutrophils for enhanced production of leukotriene B4. J Clin Invest. 1989;83(3):970-977.

37. Colas RA, Shinohara M, Dalli J, Chiang N, Serhan CN. Identification and signature profiles for pro-resolving and inflammatory lipid mediators in human tissue. Am J Physiol, Cell Physiol. 2014;307(1):C39-C54.

38. Skarke C, et al. Bioactive products formed in humans from fish oils. J Lipid Res. 2015;56(9):1808-1820.

39. Ahluwalia N, et al. Inhibited aortic aneurysm formation in BLT1-deficient mice. J Immunol. 2007;179(1):691-697.

40. Wang M, et al. Microsomal prostaglandin E synthase-1 deletion suppresses oxidative stress and angiotensin II-induced abdominal aortic aneurysm formation. Circulation. 2008;117(10):1302-1309.

41. Zhao L, et al. The 5-lipoxygenase pathway promotes pathogenesis of hyperlipidemia-dependent aortic aneurysm. Nat Med. 2004;10(9):966-973.

42. Ferreri NR, Howland WC, Stevenson DD, Spiegelberg HL. Release of leukotrienes, prostaglandins, and histamine into nasal secretions of aspirin-sensitive asthmatics during reaction to aspirin. Am Rev Respir Dis. 1988;137(4):847-854.

43. Kumlin M, Dahlén B, Björck T, Zetterström O, Granström E, Dahlén SE. Urinary excretion of leukotriene E4 and 11-dehydro-thromboxane B2 in response to bronchial provocations with allergen, aspirin, leukotriene D4, and histamine in asthmatics. Am Rev Respir Dis. 1992;146(1):96-103.

44. Noguchi K, Okubo M. Leukotrienes in nociceptive pathway and neuropathic/inflammatory pain. Biol Pharm Bull. 2011;34(8):1163-1169.

45. Yousefi B, Jadidi-Niaragh F, Azizi G, Hajighasemi F, Mirshafiey A. The role of leukotrienes in immunopathogenesis of rheumatoid arthritis [published online ahead of print March 26, 2013]. Mod Rheumatol. doi: 10.1007/s10165-013-0861-8.

46. Burnett BP, Levy RM. 5-Lipoxygenase metabolic contributions to NSAID-induced organ toxicity. Adv Ther. 2012;29(2):79-98. 
47. Li X, et al. Differential impairment of aspirin-dependent platelet cyclooxygenase acetylation by nonsteroidal antiinflammatory drugs. Proc Natl Acad Sci U S A. 2014;111(47):16830-16835.

48. Penning TD, et al. Synthesis and biological evaluation of the 1,5-diarylpyrazole class of cyclooxygenase-2 inhibitors: identification of 4-[5-(4-methylphenyl)-3-(trifluoromethyl)-1H-pyrazol-1-yl]benze nesulfonamide (SC-58635, celecoxib). J Med Chem. 1997;40(9):1347-1365.

49. Cryer B, Feldman M. Cyclooxygenase-1 and cyclooxygenase-2 selectivity of widely used nonsteroidal anti-inflammatory drugs. Am J Med. 1998;104(5):413-421.

50. Bingham S, et al. The cyclooxygenase-2 inhibitor GW406381X [2-(4-ethoxyphenyl)-3-[4-(methylsulfonyl)phenyl]-pyrazolo[1,5-b]pyridazine] is effective in animal models of neuropathic pain and central sensitization. J Pharmacol Exp Ther. 2005;312(3):1161-1169.

51. Xu D, et al. MF63 [2-(6-chloro-1H-phenanthro[9,10-d]imidazol-2-yl)-isophthalonitrile], a selective microsomal prostaglandin E synthase-1 inhibitor, relieves pyresis and pain in preclinical models of inflammation. J Pharmacol Exp Ther. 2008;326(3):754-763.

52. Murase A, Taniguchi Y, Tonai-Kachi H, Nakao K, Takada J. In vitro pharmacological characterization of CJ-042794, a novel, potent, and selective prostaglandin EP(4) receptor antagonist. Life Sci. 2008;82(3-4):226-232.

53. Brooks CD, et al. (R)-(+)-N-[3-[5-[(4-fluorophenyl)methyl]-2-thienyl]-1-methyl- 2-propynyl]-N-hydroxyurea (ABT-761), a second-generation 5-lipoxygenase inhibitor. J Med Chem. 1995;38(24):4768-4775.

54. Brideau C, et al. Pharmacology of MK-0591 (3-[1-(4-chlorobenzyl)-3-(t-butylthio)-5-(quinolin-2-yl-methoxy)- indol-2-yl]-2,2-dimethyl propanoic acid), a potent, orally active leukotriene biosynthesis inhibitor. Can J Physiol Pharmacol. 1992;70(6):799-807.

55. Askonas LJ, Kachur JF, Villani-Price D, Liang CD, Russell MA, Smith WG. Pharmacological characterization of SC-57461A (3-[methyl[3-[4-(phenylmethyl)phenoxy]propyl]amino]propanoic acid $\mathrm{HCl}$ ), a potent and selective inhibitor of leukotriene A(4) hydrolase I: in vitro studies. J Pharmacol Exp Ther. 2002;300(2):577-582.

56. Jackson WT, et al. Pharmacologic actions of the second-generation leukotriene B4 receptor antagonist LY293111: in vitro studies. J Pharmacol Exp Ther. 1999;288(1):286-294.

57. Uğur M, Melli M. Inhibitory effect of LY 255283 on the synthesis of leukotriene B(4) and thromboxane A(2) in human peripheral blood polymorphonuclear leukocytes and monocytes. Mediators Inflamm. 1993;2(6):407-409.

58. Yokomizo T, Kato K, Hagiya H, Izumi T, Shimizu T. Hydroxyeicosanoids bind to and activate the low affinity leukotriene B4 receptor, BLT2. J Biol Chem. 2001;276(15):12454-12459.

59. Huntjens DR, Danhof M, Della Pasqua OE. Pharmacokinetic-pharmacodynamic correlations and biomarkers in the development of COX-2 inhibitors. Rheumatology (Oxford). 2005;44(7):846-859.

60. Patrignani $\mathrm{P}$, et al. Biochemical and pharmacological characterization of the cyclooxygenase activity of human blood prostaglandin endoperoxide synthases. J Pharmacol Exp Ther. 1994;271(3):1705-1712.

61. O'Sullivan MG, Chilton FH, Huggins EM, McCall CE. Lipopolysaccharide priming of alveolar macrophages for enhanced synthesis of prostanoids involves induction of a novel prostaglandin H synthase. J Biol Chem. 1992;267(21):14547-14550.

62. Lee SH, Williams MV, DuBois RN, Blair IA. Targeted lipidomics using electron capture atmospheric pressure chemical ionization mass spectrometry. Rapid Commun Mass Spectrom. 2003;17(19):2168-2176.

63. Krzywinski M, et al. Circos: an information aesthetic for comparative genomics. Genome Res. 2009;19(9):1639-1645 